\title{
A General Composite Algorithms for Solving General Equilibrium Problems and Fixed Point Problems in Hilbert Spaces
}

\author{
Rattanaporn Wangkeeree, Uthai Kamraksa, \\ and Rabian Wangkeeree
}

Department of Mathematics, Faculty of Science, Naresuan University, Phitsanulok 65000, Thailand

Correspondence should be addressed to Rabian Wangkeeree, rabianw@nu.ac.th

Received 31 October 2010; Revised 8 February 2011; Accepted 8 March 2011

Academic Editor: Yuming Shi

Copyright (c) 2011 Rattanaporn Wangkeeree et al. This is an open access article distributed under the Creative Commons Attribution License, which permits unrestricted use, distribution, and reproduction in any medium, provided the original work is properly cited.

We introduce a general composite algorithm for finding a common element of the set of solutions of a general equilibrium problem and the common fixed point set of a finite family of asymptotically nonexpansive mappings in the framework of Hilbert spaces. Strong convergence of such iterative scheme is obtained which solving some variational inequalities for a strongly monotone and strictly pseudocontractive mapping. Our results extend the corresponding recent results of Yao and Liou (2010).

\section{Introduction}

Let $C$ be a nonempty, closed, convex subset of a real Hilbert space $H$. Recall that a mapping $A: C \rightarrow H$ is called $\alpha$-inverse-strongly monotone if there exists a positive real number $\alpha$ such that $\langle A x-A y, x-y\rangle \geq \alpha\|A x-A y\|^{2}$, for all $x, y \in C$. It is clear that any $\alpha$-inversestrongly monotone mapping is monotone and $1 / \alpha$-Lipschitz continuous. Let $f: C \rightarrow H$ be a $\rho$-contraction, that is, there exists a constant $\rho \in[0,1)$ such that $\|f(x)-f(y)\| \leq \rho\|x-y\|$ for all $x, y \in C$. A mapping $S: C \rightarrow C$ is said to be nonexpansive if $\|S x-S y\| \leq\|x-y\|$ for all $x, y \in C$ and asymptotically nonexpansive [1] if there exists a sequence $\left\{k_{n}\right\} \subset[0, \infty)$ with $\lim _{n \rightarrow \infty} k_{n}=0$ such that

$$
\left\|S^{n} x-S^{n} y\right\| \leq\left(1+k_{n}\right)\|x-y\|, \quad \forall x, y \in C .
$$

Denote the set of fixed points of $S$ by $\operatorname{Fix}(S)$. For asymptotically nonexpansive self-map $S$, it is well known that Fix $(S)$ is closed and convex (see, e.g., [1]). 
The class of asymptotically nonexpansive mappings which is an important generalization of that of nonexpansive mappings was introduced by Goebel and Kirk [1]. They established that if $C$ is a nonempty, closed, convex, bounded subset of a uniformly convex Banach space $E$ and $S$ is an asymptotically nonexpansive self-mapping of $C$, then $S$ has a fixed point in $C$.

Let $A: C \rightarrow H$ be a nonlinear mapping and $\phi: C \times C \rightarrow \mathbb{R}$ a bifunction. Consider a general equilibrium problem:

$$
\text { Find } z \in C \text { such that } \phi(z, y)+\langle A z, y-z\rangle \geq 0, \quad \forall y \in C \text {. }
$$

The set of all solutions of the general equilibrium problem (1.2) is denoted by EP, that is,

$$
\mathrm{EP}=\{z \in C: \phi(z, y)+\langle A z, y-z\rangle \geq 0, \forall y \in C\}
$$

If $A=0$, then (1.2) reduces to the following equilibrium problem of finding $z \in C$ such that

$$
\phi(z, y) \geq 0, \quad \forall y \in C
$$

If $\phi=0$, then (1.2) reduces to the variational inequality problem of finding $z \in C$ such that

$$
\langle A z, y-z\rangle \geq 0, \quad \forall y \in C
$$

We note that the problem (1.2) is very general in the sense that it includes, as special cases, optimization problems, variational inequalities, minimax problems, Nash equilibrium problem in noncooperative games, and others. See, for example, [2-5].

In 2005, Combettes and Hirstoaga [6] introduced an iterative algorithm of finding the best approximation to the initial data and proved a strong convergence theorem. In 2007, by using the viscosity approximation method, S. Takahashi and W. Takahashi [7] introduced another iterative scheme for finding a common element of the set of solutions of the equilibrium problem and the set of fixed points of a nonexpansive mapping. Subsequently, algorithms constructed for solving the equilibrium problems and fixed point problems have further developed by some authors. In particular, Ceng and Yao [8] introduced an iterative scheme for finding a common element of the set of solutions of the mixed equilibrium problem (1.2) and the set of common fixed points of finitely many nonexpansive mappings. Maingé and Moudafi [9] introduced an iterative algorithm for equilibrium problems and fixed point problems. Yao et al. [10] considered an iterative scheme for finding a common element of the set of solutions of the equilibrium problem and the set of common fixed points of an infinite nonexpansive mappings. Noor et al. [11] introduced an iterative method for solving fixed point problems and variational inequality problems. Wangkeeree [12] introduced a new iterative scheme for finding the common element of the set of common fixed points of nonexpansive mappings, the set of solutions of an equilibrium problem, and the set of solutions of the variational inequality. Wangkeeree and Kamraksa [13] introduced an iterative algorithm for finding a common element of the set of solutions of a mixed equilibrium problem, the set of fixed points of an infinite family of nonexpansive mappings, and the set of solutions of a general system of variational inequalities for a cocoercive mapping in a real Hilbert space. Their results extend and improve many results in the 
literature. For some works related to the equilibrium problem, fixed point problems, and the variational inequality problem, please see [1-57] and the references therein.

However, we note that all constructed algorithms in $[7,9-13,16,57]$ do not work to find the minimum-norm solution of the corresponding fixed point problems and the equilibrium problems. Very recently, Yao and Liou [46] purposed some algorithms for finding the minimum-norm solution of the fixed point problems and the equilibrium problems. They first suggested two new composite algorithms (one implicit and one explicit) for solving the above minimization problem. To be more precisely, let $C$ be a nonempty, closed, convex subset of $H, \phi: C \times C \rightarrow \mathbb{R}$ a bifunction satisfying certain conditions, and $S: C \rightarrow C$ a nonexpansive mapping such that $\Omega:=\operatorname{Fix}(S) \cap \mathrm{EP} \neq \emptyset$. Let $f$ be a contraction on a Hilbert space $H$. For given $x_{0} \in C$ arbitrarily, let the sequence $\left\{x_{n}\right\}$ be generated iteratively by

$$
\begin{array}{cc}
\phi\left(u_{n}, y\right)+\left\langle A x_{n}, y-u_{n}\right\rangle+\frac{1}{r}\left\langle y-u_{n}, u_{n}-x_{n}\right\rangle \geq 0, & \forall y \in C, \\
x_{n+1}=\mu_{n} P_{C}\left[\alpha_{n} f\left(x_{n}\right)+\left(1-\alpha_{n}\right) S x_{n}\right]+\left(1-\mu_{n}\right) u_{n}, \quad n \geq 0
\end{array}
$$

where $A$ is an $\alpha$-inverse strongly monotone mapping. They proved that if $\left\{\alpha_{n}\right\}$ and $\left\{\mu_{n}\right\}$ are two sequences in $[0,1]$ satisfying the following conditions:

(i) $\lim _{n \rightarrow \infty} \alpha_{n}=0, \sum_{n=0}^{\infty} \alpha_{n}=\infty$ and $\lim _{n \rightarrow \infty}\left(\alpha_{n+1} / \alpha_{n}\right)=1$,

(ii) $0<\liminf _{n \rightarrow \infty} \mu_{n} \leq \lim \sup _{n \rightarrow \infty} \mu_{n}<1$ and $\lim _{n \rightarrow \infty}\left(\left(\mu_{n+1}-\mu_{n}\right) / \alpha_{n+1}\right)=0$, then, the sequence $\left\{x_{n}\right\}$ generated by (1.6) converges strongly to $x^{*} \in \Omega$ which is the unique solution of variational inequality

$$
\left\langle(I-f) x^{*}, x-x^{*}\right\rangle \geq 0, \quad x \in \Omega
$$

In particular, if we take $f=0$ in (1.6), then the sequence $\left\{x_{n}\right\}$ generated by

$$
\begin{gathered}
\phi\left(u_{n}, y\right)+\left\langle A x_{n}, y-u_{n}\right\rangle+\frac{1}{r}\left\langle y-u_{n}, u_{n}-x_{n}\right\rangle \geq 0, \quad \forall y \in C \\
x_{n+1}=\mu_{n} P_{C}\left[\left(1-\alpha_{n}\right) S x_{n}\right]+\left(1-\mu_{n}\right) u_{n}, \quad n \geq 0
\end{gathered}
$$

converges strongly to a solution of the minimization problem which is the problem of finding $x^{*}$ such that

$$
x^{*}=\arg \min _{x \in \Omega}\|x\|^{2},
$$

where $\Omega$ stands for the intersection set of the solution set of the general equilibrium problem and the fixed points set of a nonexpansive mapping.

On the other hand, iterative approximation methods for nonexpansive mappings have recently been applied to solve convex minimization problems; see, for example, [25, 43, 44] and the references therein. Let $B$ be a strongly positive bounded linear operator on $H$, that is, there is a constant $\bar{\gamma}>0$ with property

$$
\langle B x, x\rangle \geq \bar{\gamma}\|x\|^{2} \quad \forall x \in H .
$$


A typical problem is to minimize a quadratic function over the set of the fixed points of a nonexpansive mapping on a real Hilbert space $H$

$$
\min _{x \in \operatorname{Fix}(S)} \frac{1}{2}\langle B x, x\rangle-\langle x, b\rangle
$$

where $b$ is a given point in $H$. In 2003, Xu [43] proved that the sequence $\left\{x_{n}\right\}$ defined by the iterative method below, with the initial guess $x_{0} \in H$ chosen arbitrarily:

$$
x_{n+1}=\left(I-\alpha_{n} B\right) T x_{n}+\alpha_{n} u, \quad n \geq 0,
$$

converges strongly to the unique solution of the minimization problem (1.11) provided the sequence $\left\{\alpha_{n}\right\}$ satisfies certain conditions. Using the viscosity approximation method, Moudafi [29] introduced the following iterative process for nonexpansive mappings (see [43] for further developments in both Hilbert and Banach spaces). Let $f$ be a contraction on $H$. Starting with an arbitrary initial $x_{0} \in H$, define a sequence $\left\{x_{n}\right\}$ recursively by

$$
x_{n+1}=\left(1-\alpha_{n}\right) T x_{n}+\alpha_{n} f\left(x_{n}\right), \quad n \geq 0,
$$

where $\left\{\alpha_{n}\right\}$ is a sequence in $(0,1)$. It is proved $[29,43]$ that under certain appropriate conditions imposed on $\left\{\alpha_{n}\right\}$, the sequence $\left\{x_{n}\right\}$ generated by (1.13) strongly converges to the unique solution $x^{*}$ in $C$ of the variational inequality

$$
\left\langle(I-f) x^{*}, x-x^{*}\right\rangle \geq 0, \quad x \in H .
$$

Recently, Marino and $\mathrm{Xu}$ [28] mixed the iterative method (1.12) and the viscosity approximation method (1.13) introduced by Moudafi [29] and considered the following general iterative method:

$$
x_{n+1}=\left(I-\alpha_{n} B\right) T x_{n}+\alpha_{n} \gamma f\left(x_{n}\right), \quad n \geq 0,
$$

where $B$ is a strongly positive bounded linear operator on $H$. They proved that if the sequence $\left\{\alpha_{n}\right\}$ of parameters satisfies the certain conditions, then the sequence $\left\{x_{n}\right\}$ generated by (1.15) converges strongly to the unique solution $x^{*}$ in $H$ of the variational inequality

$$
\left\langle(B-r f) x^{*}, x-x^{*}\right\rangle \geq 0, \quad x \in H
$$

which is the optimality condition for the minimization problem: $\min _{x \in \operatorname{Fix}(S)}(1 / 2)\langle B x, x\rangle-$ $h(x)$, where $h$ is a potential function for $\gamma f$ (i.e., $h^{\prime}(x)=\gamma f(x)$ for $x \in H$ ).

Recall that a mapping $F: H \rightarrow H$ is called $\delta$-strongly monotone if there exists a positive constant $\delta$ such that

$$
\langle F x-F y, x-y\rangle \geq \delta\|x-y\|^{2}, \quad \forall x, y \in H .
$$


Recall also that a mapping $F$ is called $\lambda$-strictly pseudocontractive if there exists a positive constant $\lambda$ such that

$$
\langle F x-F y, x-y\rangle \leq\|x-y\|^{2}-\lambda\|(x-y)-(F x-F y)\|^{2}, \quad \forall x, y \in H .
$$

It is easy to see that (1.18) can be rewritten as

$$
\langle(I-F) x-(I-F) y, x-y\rangle \geq \lambda\|(I-F) x-(I-F) y\|^{2} .
$$

Remark 1.1. If $F$ is a strongly positive bounded linear operator on $H$ with coefficient $\bar{\gamma}$, then $F$ is $\bar{\gamma}$-strongly monotone and 12 -strictly pseudocontractive. In fact, since $F$ is a strongly positive, bounded, linear operator with coefficient $\bar{\gamma}$, we have

$$
\langle F x-F y, x-y\rangle=\langle F(x-y), x-y\rangle \geq \bar{\gamma}\|x-y\|^{2} .
$$

Therefore, $F$ is $\bar{\gamma}$-strongly monotone. On the other hand,

$$
\begin{aligned}
\|(I-F) x-(I-F) y\|^{2} & =\langle(x-y)-(F x-F y),(x-y)-(F x-F y)\rangle \\
& =\langle x-y, x-y\rangle-2\langle F x-F y, x-y\rangle+\langle F x-F y, F x-F y\rangle \\
& =\|x-y\|^{2}-2\langle F x-F y, x-y\rangle+\|F x-F y\|^{2} \\
& \leq\|x-y\|^{2}-2\langle F x-F y, x-y\rangle+\|F\|^{2}\|x-y\|^{2} .
\end{aligned}
$$

Since $F$ is strongly positive if and only if $(1 /\|F\|) F$ is strongly positive, we may assume, without loss of generality, that $\|F\|=1$. From (1.21), we have

$$
\begin{aligned}
\langle F x-F y, x-y\rangle & \leq\|x-y\|^{2}-\frac{1}{2}\|(I-F) x-(I-F) y\|^{2} \\
& =\|x-y\|^{2}-\frac{1}{2}\|(x-y)-(F x-F y)\|^{2} .
\end{aligned}
$$

Hence, $F$ is 12-strictly pseudocontractive.

In this paper, motivated by the above results, we introduce a general iterative scheme below in a real Hilbert space $H$, with the initial guess $x_{0} \in C$ chosen arbitrary:

$$
\begin{gathered}
\phi\left(u_{n}, y\right)+\left\langle A x_{n}, y-u_{n}\right\rangle+\frac{1}{r}\left\langle y-u_{n}, u_{n}-x_{n}\right\rangle \geq 0, \quad \forall y \in C, \\
y_{n}=\alpha_{n} \gamma f\left(x_{n}\right)+\left(I-\alpha_{n} F\right) S_{i(n+1)}^{p(n+1)} x_{n} \\
x_{n+1}=\mu_{n} P_{C}\left[y_{n}\right]+\left(1-\mu_{n}\right) u_{n}, \quad n \geq 0
\end{gathered}
$$

where $p(n)=j+1$ if $j N<n \leq(j+1) N, j=1,2, \ldots$ and $n=j N+i(n), i(n) \in\{1,2, \ldots, N\}$, $C$ is a nonempty, closed, convex subset of $H,\left\{\alpha_{n}\right\}$ and $\left\{\mu_{n}\right\}$ are two sequences in $[0,1]$, 
$\phi: C \times C \rightarrow \mathbb{R}$ is a bifunction satisfying certain conditions, $S_{1}, S_{2}, \ldots, S_{N}: C \rightarrow C$ is a finite

family of asymptotically nonexpansive mappings with sequences $\left\{1+k_{p(n)}^{i(n)}\right\}$, respectively, $f: C \rightarrow H$ is a contraction with coefficient $0<\rho<1, F$ is $\delta$-strongly monotone and $\lambda$ strictly pseudocontractive with $\delta+\lambda>1, \gamma$ is a positive real number such that $\gamma<(1 / \rho)$ $(1-\sqrt{(1-\delta) / \lambda})$, and $A$ is an $\alpha$-inverse strongly monotone mapping. We prove that the proposed algorithm converges strongly to $x^{*} \in \Omega$ which is the unique solution of the following variational inequality:

$$
\left\langle(F-\gamma f) x^{*}, x-x^{*}\right\rangle \geq 0, \quad x \in \Omega
$$

In particular,

(I) if $F$ is a strongly positive bounded linear operator on $H$, then $x^{*}$ is the unique solution of the variational inequality (1.16),

(II) if $F=I$, the identity mapping on $H$ and $\gamma=1$, then $x^{*}$ is the unique solution of the variational inequality (1.14),

(III) if $F=I$, the identity mapping on $H$ and $f=0$, then $x^{*}$ is the unique solution of minimization problem (1.9).

The results presented in this paper extend and improve the main results in Yao and Liou [46], Marino and Xu [28], and many others.

\section{Preliminaries}

Let $C$ be a nonempty, closed, convex subset of a real Hilbert space $H$. For every point $x \in H$, there exists a unique nearest point in $C$, denoted by $P_{C} x$ such that

$$
\left\|x-P_{C} x\right\| \leq\|x-y\|, \quad \forall y \in C .
$$

$P_{C}$ is called the metric projection of $H$ onto $C$. It is well known that $P_{C}$ is a nonexpansive mapping of $H$ onto $C$ and satisfies

$$
\left\langle x-y, P_{C} x-P_{C} y\right\rangle \geq\left\|P_{C} x-P_{C} y\right\|^{2},
$$

for every $x, y \in H$. Moreover, $P_{C} x$ is characterized by the following properties: $P_{C} x \in C$ and

$$
\begin{gathered}
\left\langle x-P_{C} x, y-P_{C} x\right\rangle \leq 0, \\
\|x-y\|^{2} \geq\left\|x-P_{C} x\right\|^{2}+\left\|y-P_{C} x\right\|^{2},
\end{gathered}
$$

for all $x \in H, y \in C$. For more details, see [39]. We will make use of the following well-known result.

Lemma 2.1. Let $H$ be a Hilbert space. Then, the following inequality holds:

$$
\|x+y\|^{2} \leq\|x\|^{2}+2\langle y, x+y\rangle, \quad \forall x, y \in H .
$$


Throughout this paper, we assume that a bifunction $\phi: C \times C \rightarrow \mathbb{R}$ satisfies the following conditions:

(A1) $\phi(x, x)=0$ for all $x \in C$,

(A2) $\phi$ is monotone, that is, $\phi(x, y)+\phi(y, x) \leq 0$ for all $x, y \in C$,

(A3) for each $x, y, z \in C, \lim _{t \downarrow 0} \phi(t z+(1-t) x, y) \leq \phi(x, y)$,

(A4) for each $x \in C$, the mapping $y \mapsto \phi(x, y)$ is convex and lower semicontinuous.

We need the following lemmas for proving our main results.

Lemma 2.2 (see [6]). Let $C$ be a nonempty, closed, convex subset of a real Hilbert space $H$. Let $\phi: C \times C \rightarrow \mathbb{R}$ be a bifunction which satisfies conditions (A1)-(A4). Let $r>0$ and $x \in C$. Then, there exists $z \in C$ such that

$$
\phi(z, y)+\frac{1}{r}\langle y-z, z-x\rangle \geq 0, \quad \forall y \in C .
$$

Further, if $T_{r}(x)=\{z \in C: \phi(z, y)+(1 / r)\langle y-z, z-x\rangle \geq 0, \forall y \in C\}$, then the following hold:

(i) $T_{r}$ is single-valued and $T_{r}$ is firmly nonexpansive, that is, for any $x, y \in H$,

$$
\left\|T_{r} x-T_{r} y\right\|^{2} \leq\left\langle T_{r} x-T_{r} y, x-y\right\rangle
$$

(ii) $\mathrm{EP}$ is closed and convex and $\mathrm{EP}=\operatorname{Fix}\left(T_{r}\right)$.

Lemma 2.3 (see [30]). Let $C$ be a nonempty, closed, convex subset of a real Hilbert space $H$. Let the mapping $A: C \rightarrow H$ be $\alpha$-inverse strongly monotone and $r>0$ a constant. Then, one has

$$
\|(I-r A) x-(I-r A) y\|^{2} \leq\|x-y\|^{2}+r(r-2 \alpha)\|A x-A y\|^{2}, \quad \forall x, y \in C .
$$

In particular, if $0 \leq r \leq 2 \alpha$, then $I-r A$ is nonexpansive.

Lemma 2.4 (see [45]). Let $S$ be an asymptotically nonexpansive mapping defined on a bounded, closed, convex subset $C$ of a Hilbert space $H$. If $\left\{x_{n}\right\}$ is a sequence in $C$ such that $x_{n} \rightarrow x$ and $\left\|S x_{n}-x_{n}\right\| \rightarrow 0$ as $n \rightarrow \infty$, then $x \in \operatorname{Fix}(S)$.

Lemma 2.5 (see [44]). Assume $\left\{a_{n}\right\}$ is a sequence of nonnegative real numbers such that

$$
a_{n+1} \leq\left(1-\alpha_{n}\right) a_{n}+\alpha_{n} \sigma_{n}+\gamma_{n}, \quad n \geq 0,
$$

where $\left\{\alpha_{n}\right\},\left\{\sigma_{n}\right\}$, and $\left\{\gamma_{n}\right\}$ are nonnegative real sequences satisfying the following conditions:

(i) $\left\{\alpha_{n}\right\} \subset[0,1], \sum_{n=1}^{\infty} \alpha_{n}=\infty$,

(ii) $\limsup _{n \rightarrow \infty} \sigma_{n} \leq 0$,

(iii) $\sum_{n=1}^{\infty} \gamma_{n}<\infty$.

Then, $\lim _{n \rightarrow \infty} a_{n}=0$. 
Lemma 2.6 (see [41]). Let E be a strictly convex Banach space and $C$ a closed, convex subset of $E$. Let $S_{1}, S_{2}, \ldots, S_{N}: C \rightarrow C$ be a finite family of nonexpansive mappings of $C$ into itself such that the set of common fixed points of $S_{1}, S_{2}, \ldots, S_{N}$ is nonempty. Let $T_{1}, T_{2}, \ldots, T_{N}: C \rightarrow C$ be mappings given by

$$
T_{i}=\left(1-\alpha_{i}\right) I+\alpha_{i} S_{i}, \quad \forall i=1,2, \ldots, N,
$$

where I denotes the identity mapping on $C$. Then, the finite family $\left\{T_{1}, T_{2}, \ldots, T_{N}\right\}$ satisfies the following:

$$
\begin{gathered}
\bigcap_{i=1}^{N} \operatorname{Fix}\left(T_{i}\right)=\bigcap_{i=1}^{N} \operatorname{Fix}\left(S_{i}\right), \\
\bigcap_{i=1}^{N} \operatorname{Fix}\left(T_{i}\right)=\operatorname{Fix}\left(T_{N} T_{N-1} T_{N-2} \cdots T_{1}\right)=\operatorname{Fix}\left(T_{1} T_{N} \cdots T_{2}\right)=\operatorname{Fix}\left(T_{N-1} T_{N-2} \cdots T_{1} T_{N}\right) .
\end{gathered}
$$

The following lemma can be found in [35, Lemma 2.7]. For the sake of the completeness, we include its proof in a Hilbert space's version.

Lemma 2.7. Let $H$ be a real Hilbert space and $F: H \rightarrow H$ a mapping.

(i) If $F$ is $\delta$-strongly monotone and $\lambda$-strictly pseudocontractive with $\delta+\lambda>1$, then $I-F$ is contractive with constant $\sqrt{(1-\delta) / \lambda}$.

(ii) If $F$ is $\delta$-strongly monotone and $\lambda$-strictly pseudocontractive with $\delta+\lambda>1$, then for any fixed number $\tau \in(0,1), I-\tau F$ is contractive with constant $1-\tau(1-\sqrt{(1-\delta) / \lambda})$.

Proof. (i) For any $x, y \in H$, we have

$$
\lambda\|(I-F) x-(I-F) y\|^{2} \leq\|x-y\|^{2}-\langle F x-F y, x-y\rangle \leq(1-\delta)\|x-y\|^{2}, \quad \forall x, y \in H .
$$

Thus,

$$
\|(I-F) x-(I-F) y\| \leq \sqrt{\frac{1-\delta}{\lambda}}\|x-y\|, \quad \forall x, y \in H
$$

Since $\delta+\lambda>1$, we have $(1-\delta) / \lambda \in(0,1)$. Hence, $I-F$ is contractive with constant $\sqrt{(1-\delta) / \lambda}$.

(ii) Since $I-F$ is contractive with constant $\sqrt{(1-\delta) / \lambda}$, we have for any $\tau \in(0,1)$,

$$
\begin{aligned}
\|x-y-\tau(F x-F y)\| & =\|(1-\tau)(x-y)+\tau[(I-F) x-(I-F) y]\| \\
& \leq(1-\tau)\|x-y\|+\tau\|(I-F) x-(I-F) y\|
\end{aligned}
$$


Abstract and Applied Analysis

$$
\begin{aligned}
& \leq(1-\tau)\|x-y\|+\tau \sqrt{\frac{1-\delta}{\lambda}}\|x-y\| \\
& =\left(1-\tau\left(1-\sqrt{\frac{1-\delta}{\lambda}}\right)\right)\|x-y\|, \quad \forall x, y \in H .
\end{aligned}
$$

Hence, $I-\tau F$ is contractive with constant $1-\tau(1-\sqrt{(1-\delta) / \lambda})$.

Lemma 2.8. Let $S_{1}, S_{2}, \ldots, S_{N}: C \rightarrow C$ be a finite family of asymptotically nonexpansive mappings with sequences $\left\{1+k_{p(n)}^{i(n)}\right\}$, respectively, such that $k_{p(n)}^{i(n)} \rightarrow 0$ as $n \rightarrow \infty$. Then, there exists a sequence $\left\{h_{n}\right\} \subset[0, \infty)$ with $h_{n} \rightarrow 0$ as $n \rightarrow \infty$ such that

$$
\left\|S_{i(n)}^{p(n)} x-S_{i(n)}^{p(n)} y\right\| \leq\left(1+h_{n}\right)\|x-y\|, \quad \forall x, y \in C,
$$

where $p(n)=j+1$ if $j N<n \leq(j+1) N, j=1,2, \ldots$ and $n=j N+i(n) ; i(n) \in\{1,2, \ldots, N\}$.

Proof. Define the sequence $\left\{h_{n}\right\}$ by $h_{n}:=\max \left\{k_{p(n)}^{i(n)}: 1 \leq i(n) \leq N\right\}$ and the result follows immediately.

In the rest of our discussion in this paper, we will assume that $p(n)=j+1$ if $j N<n \leq$ $(j+1) N, j=1,2, \ldots$ and $n=j N+i(n) ; i(n) \in\{1,2, \ldots, N\}$ and $h_{n}:=\max \left\{k_{p(n)}^{i(n)}: 1 \leq i(n) \leq N\right\}$ for all $n \geq 1$, and for each $n \geq 1, n=(p(n)-1) N+i(n)$.

\section{Main Results}

Now, we are a position to state and prove our main results.

Theorem 3.1. Let $C$ be a nonempty, closed, convex subset of a real Hilbert space $H$. Let $S_{1}, S_{2}, \ldots$, $S_{N}: C \rightarrow C$ be a finite family of asymptotically nonexpansive mappings with sequences $\left\{1+k_{p(n)}^{i(n)}\right\}$, respectively, such that $k_{p(n)}^{i(n)} \rightarrow 0$ as $n \rightarrow \infty, h_{n}:=\max _{1 \leq i(n) \leq N}\left\{k_{p(n)}^{i(n)}\right\}$ and $\Gamma:=\bigcap_{i=1}^{N} \operatorname{Fix}\left(S_{i}\right)$,

$$
\Gamma=\operatorname{Fix}\left(S_{N} S_{N-1} S_{N-2} \cdots S_{1}\right)=\operatorname{Fix}\left(S_{1} S_{N} \cdots S_{2}\right)=\cdots=\operatorname{Fix}\left(S_{N-1} S_{N-2} \cdots S_{1} S_{N}\right) .
$$

Let $A: C \rightarrow H$ be an $\alpha$-inverse strongly monotone mapping. Let $\phi: C \times C \rightarrow \mathbb{R}$ be a bifunction which satisfies conditions (A1)-(A4) such that $\Omega:=\mathrm{EP} \cap \Gamma$ is nonempty. Let $F: C \rightarrow H$ be $\delta$ strongly monotone and $\lambda$-strictly pseudocontractive with $\delta+\lambda>1, f: C \rightarrow H$ a $\rho$-contraction, $\gamma$ a positive real number such that $\gamma<(1-\sqrt{(1-\delta) / \lambda}) / \rho$, and $r$ a constant such that $r \in(0,2 \alpha)$. For $x_{0} \in C$ arbitrarily, let the sequence $\left\{x_{n}\right\}$ be generated iteratively by (1.23). Suppose that $\left\{\alpha_{n}\right\}$ and $\left\{\mu_{n}\right\}$ are two sequences in $[0,1]$ satisfying the following conditions:

(C1) $\lim _{n \rightarrow \infty} \alpha_{n}=0, \lim _{n \rightarrow \infty}\left(\alpha_{n+1} / \alpha_{n}\right)=1, \sum_{n=0}^{\infty} \alpha_{n}=\infty$ and $\lim _{n \rightarrow \infty}\left(h_{n} / \alpha_{n}\right)=0$,

(C2) $0<\liminf _{n \rightarrow \infty} \mu_{n} \leq \lim \sup _{n \rightarrow \infty} \mu_{n}<1$ and $\lim _{n \rightarrow \infty}\left(\left(\mu_{n+1}-\mu_{n}\right) / \alpha_{n+1}\right)=0$. 
Assume that $\sum_{n=1}^{\infty} \sup _{z \in B}\left\|S_{i(n+1)}^{p(n+1)} z-S_{i(n)}^{p(n)} z\right\|<\infty$, for each bounded subset $B$ of $C$. Then, the sequence $\left\{x_{n}\right\}$ converges strongly to $x^{*}$ of the following variational inequality:

$$
\left\langle(F-\gamma f) x^{*}, x-x^{*}\right\rangle \geq 0, \quad x \in \Omega
$$

or equivalently $\tilde{x}=P_{\Omega}(I-F+\gamma f) \tilde{x}$, where $P_{\Omega}$ is the metric projection of $H$ onto $\Omega$.

Proof. First, we rewrite the sequence $\left\{x_{n}\right\}$ by the following:

$$
x_{n+1}=\mu_{n} P_{C}\left[y_{n}\right]+\left(1-\mu_{n}\right) T_{r}\left(x_{n}-r A x_{n}\right), \quad n \geq 0,
$$

where the mapping $T_{r}$ is defined in Lemma 2.2. Pick $z \in \Omega$ and $u_{n}=T_{r}\left(x_{n}-r A x_{n}\right)$. The nonexpansivity of $T_{r}$ and $I-r A$ implies that

$$
\begin{aligned}
\left\|u_{n}-z\right\| & =\left\|T_{r}\left(x_{n}-r A x_{n}\right)-T_{r}(z-r A z)\right\| \\
& \leq\left\|x_{n}-z\right\|, \quad \forall z \in \Omega .
\end{aligned}
$$

Setting $\bar{\gamma}:=(1-\sqrt{(1-\delta) / \lambda})$ and using Lemma 2.7(ii), we have

$$
\begin{aligned}
\left\|y_{n}-z\right\| & =\left\|\alpha_{n} \gamma\left(f\left(x_{n}\right)-F z\right)+\left(I-\alpha_{n} F\right)\left(S_{i(n+1)}^{p(n+1)} x_{n}-z\right)\right\| \\
& \leq \alpha \alpha_{n} \gamma\left\|x_{n}-z\right\|+\alpha_{n}\left\|\gamma f\left(x_{n}\right)-F z\right\|+\left(1-\alpha_{n} \bar{\gamma}\right)\left(1+h_{n+1}\right)\left\|x_{n}-z\right\| \\
& =\left[1-\alpha_{n}(\bar{\gamma}-\alpha \gamma)+\left(1-\alpha_{n} \bar{\gamma}\right) h_{n+1}\right]\left\|x_{n}-z\right\|+\alpha_{n}\left\|\gamma f\left(x_{n}\right)-F z\right\| .
\end{aligned}
$$

By our assumptions, we have $\left(1-\alpha_{n} \bar{\gamma}\right)\left(h_{n+1} / \alpha_{n}\right) \rightarrow 0$ as $n \rightarrow \infty$. We can assume, without loss of generality, that $\left(1-\alpha_{n} \bar{\gamma}\right)\left(h_{n+1} / \alpha_{n}\right)<(1 / 2)(\bar{\gamma}-\alpha \gamma)$. Applying Lemma 2.7, we can calculate the following:

$$
\begin{aligned}
\left\|x_{n+1}-z\right\|= & \left\|\mu_{n}\left(P_{C}\left[y_{n}\right]-z\right)+\left(1-\mu_{n}\right)\left(u_{n}-z\right)\right\| \\
\leq & \mu_{n}\left\|P_{C}\left[y_{n}\right]-z\right\|+\left(1-\mu_{n}\right)\left\|u_{n}-z\right\| \\
\leq & \mu_{n}\left\|y_{n}-z\right\|+\left(1-\mu_{n}\right)\left\|x_{n}-z\right\| \\
\leq & \mu_{n}\left[1-\alpha_{n}(\bar{\gamma}-\alpha \gamma)+\left(1-\alpha_{n} \bar{\gamma}\right) h_{n+1}\right]\left\|x_{n}-z\right\| \\
& +\mu_{n} \alpha_{n}\left\|\gamma f\left(x_{n}\right)-F z\right\|+\left(1-\mu_{n}\right)\left\|x_{n}-z\right\| \\
= & {\left[1-\mu_{n} \alpha_{n}\left[(\bar{\gamma}-\alpha \gamma)-\left(1-\alpha_{n} \bar{\gamma}\right) \frac{h_{n+1}}{\alpha_{n}}\right]\right]\left\|x_{n}-z\right\|+\mu_{n} \alpha_{n}\left\|\gamma f\left(x_{n}\right)-F z\right\| } \\
\leq & {\left[1-\frac{1}{2} \mu_{n} \alpha_{n}(\bar{\gamma}-\alpha \gamma)\right]\left\|x_{n}-z\right\|+\frac{\mu_{n} \alpha_{n}(1 / 2)(\bar{\gamma}-\alpha \gamma)}{(1 / 2)(\bar{\gamma}-\alpha \gamma)}\left\|\gamma f\left(x_{n}\right)-F z\right\| . }
\end{aligned}
$$


By induction, we obtain, for all $n \geq 0$,

$$
\left\|x_{n}-z\right\| \leq \max \left\{\left\|x_{0}-z\right\|, \frac{2\left\|\gamma f\left(x_{0}\right)-F(z)\right\|}{\bar{\gamma}-\alpha \gamma}\right\} .
$$

Hence, $\left\{x_{n}\right\}$ is bounded. Consequently, we deduce that $\left\{u_{n}\right\},\left\{f\left(x_{n}\right)\right\}$, and $\left\{y_{n}\right\}$ are all bounded.

Next, we show that

$$
\lim _{n \rightarrow \infty}\left\|x_{n+N}-x_{n}\right\|=0 .
$$

From (1.23), we have

$$
\begin{aligned}
\left\|y_{n+N}-y_{n+N-1}\right\|= & \| \alpha_{n+N} \gamma f\left(x_{n+N}\right)+\left(I-\alpha_{n+N} F\right) S_{i(n+N+1)}^{p(n+N+1)} x_{n+N} \\
& -\alpha_{n+N-1} \gamma f\left(x_{n+N-1}\right)-\left(I-\alpha_{n+N-1} F\right) S_{i(n+N)}^{p(n+N)} x_{n+N-1} \| \\
= & \| \alpha_{n+N} \gamma\left(f\left(x_{n+N}\right)-f\left(x_{n+N-1}\right)\right)+\left(\alpha_{n+N}-\alpha_{n+N-1}\right) \gamma f\left(x_{n+N-1}\right) \\
& +\left(I-\alpha_{n+N} F\right)\left(S_{i(n+N+1)}^{p(n+N+1)} x_{n+N}-S_{i(n+N+1)}^{p(n+N+1)} x_{n+N-1}\right) \\
& +\left[\left(I-\alpha_{n+N} F\right)-\left(I-\alpha_{n+N-1} F\right)\right] S_{i(n+N+1)}^{p(n+N+1)} x_{n+N-1} \\
& +\left(I-\alpha_{n+N-1} F\right)\left(S_{i(n+N+1)}^{p(n+N+1)} x_{n+N-1}-S_{i(n+N)}^{p(n+N)} x_{n+N-1}\right) \| \\
\leq & \alpha_{n+N} \gamma \alpha\left\|x_{n+N}-x_{n+N-1}\right\|+\left|\alpha_{n+N}-\alpha_{n+N-1}\right| \gamma\left\|f\left(x_{n+N-1}\right)\right\| \\
& +\left(1-\alpha_{n+N} \bar{\gamma}\right)\left(1+h_{n+N+1}\right)\left\|x_{n+N}-x_{n+N-1}\right\| \\
& +\left|\alpha_{n+N-1}-\alpha_{n+N}\right|\|F\|\left\|S_{i(n+N+1)}^{p(n+N+1)} x_{n+N-1}\right\| \\
& +\left(1-\alpha_{n+N-1} \bar{\gamma}\right)\left\|S_{i(n+N+1)}^{p(n+N+1)} x_{n+N-1}-S_{i(n+N)}^{p(n+N)} x_{n+N-1}\right\| \\
\leq & \alpha_{n+N} \gamma \alpha\left\|x_{n+N}-x_{n+N-1}\right\|+\left|\alpha_{n+N}-\alpha_{n+N-1}\right| \gamma\left\|f\left(x_{n+N-1}\right)\right\| \\
& +\left(1-\alpha_{n+N} \bar{\gamma}\right)\left(1+h_{n+N+1}\right)\left\|x_{n+N}-x_{n+N-1}\right\| \\
& +\left|\alpha_{n+N-1}-\alpha_{n+N}\right|\|F\|\left\|S_{i(n+N+1)}^{p(n+N+1)} x_{n+N-1}\right\| \\
& +\sup _{x \in\left\{x_{n}: n \in \mathbb{N}\right\}}\left\|S_{i(n+N+1)}^{p(n+N+1)} x-S_{i(n+N)}^{p(n+N)} x\right\| \\
& \\
&
\end{aligned}
$$


and from (3.3), we have

$$
\begin{aligned}
\left\|x_{n+N+1}-x_{n+N}\right\|= & \| \mu_{n+N} P_{C}\left[y_{n+N}\right]+\left(1-\mu_{n+N}\right) u_{n+N}-\mu_{n+N-1} P_{C}\left[y_{n+N-1}\right] \\
& \quad-\left(1-\mu_{n+N-1}\right) u_{n+N-1} \| \\
= & \| \mu_{n+N}\left(P_{C}\left[y_{n+N}\right]-P_{C}\left[y_{n+N-1}\right]\right)+\left(\mu_{n+N}-\mu_{n+N-1}\right) P_{C}\left[y_{n+N-1}\right] \\
& \quad+\left(1-\mu_{n+N}\right)\left(u_{n+N}-u_{n+N-1}\right)+\left(\mu_{n+N-1}-\mu_{n+N}\right) u_{n+N-1} \| \\
\leq & \mu_{n+N}\left\|y_{n+N}-y_{n+N-1}\right\|+\left(1-\mu_{n+N}\right)\left\|u_{n+N}-u_{n+N-1}\right\| \\
& +\left|\mu_{n+N}-\mu_{n+N-1}\right|\left(\left\|P_{C}\left[y_{n+N-1}\right]\right\|+\left\|u_{n+N-1}\right\|\right) \\
\left\|u_{n+N}-u_{n+N-1}\right\|= & \left\|T_{r}\left(x_{n+N}-r A x_{n+N}\right)-T_{r}\left(x_{n+N-1}-r A x_{n+N-1}\right)\right\| \\
\leq & \left\|\left(x_{n+N}-r A x_{n+N}\right)-\left(x_{n+N-1}-r A x_{n+N-1}\right)\right\| \\
\leq & \left\|x_{n+N}-x_{n+N-1}\right\| .
\end{aligned}
$$

Therefore,

$$
\begin{aligned}
\left\|x_{n+N+1}-x_{n+N}\right\| \leq & \mu_{n+N} \alpha_{n+N} \gamma \alpha\left\|x_{n+N}-x_{n+N-1}\right\|+\mu_{n+N}\left|\alpha_{n+N}-\alpha_{n+N-1}\right| \gamma\left\|f\left(x_{n+N-1}\right)\right\| \\
& +\mu_{n+N}\left(1-\alpha_{n+N} \bar{\gamma}\right)\left(1+h_{n+N+1}\right)\left\|x_{n+N}-x_{n+N-1}\right\| \\
& +\mu_{n+N}\left|\alpha_{n+N-1}-\alpha_{n+N}\right|\|F\|\left\|S_{i(n+N+1)}^{p(n+N+1)} x_{n+N-1}\right\| \\
& +\mu_{n+N} \sup _{x \in\left\{x_{n}: n \in \mathbb{N}\right\}}\left\|S_{i(n+N+1)}^{p(n+N+1)} x-S_{i(n+N)}^{p(n+N)} x\right\| \\
& +\left(1-\mu_{n+N}\right)\left\|x_{n+N}-x_{n+N-1}\right\| \\
& +\left|\mu_{n+N}-\mu_{n+N-1}\right|\left(\left\|P_{C}\left[y_{n+N-1}\right]\right\|+\left\|u_{n+N-1}\right\|\right) \\
\leq & \left(1-\mu_{n+N} \alpha_{n+N}(\bar{\gamma}-\gamma \alpha)\right)\left\|x_{n+N}-x_{n+N-1}\right\|+\mu_{n+N} \alpha_{n+N} \\
& \times\left[\left(\frac{h_{n+N+1}}{\alpha_{n+N}}+h_{n+N+1} \bar{\gamma}\right) M\right. \\
& +\left|1-\frac{\alpha_{n+N-1}}{\alpha_{n+N}}\right| \gamma\left\|f\left(x_{n+N-1}\right)\right\|+\left|\frac{\alpha_{n+N-1}}{\alpha_{n+N}}-1\right|\|F\|\left\|S_{i(n+N+1)}^{p(n+N+1)} x_{n+N-1}\right\| \\
& \left.\quad+\frac{1}{\mu_{n+N}}\left|\frac{\mu_{n+N}-\mu_{n+N-1}}{\alpha_{n+N}}\right|\left(\left\|P_{C}\left[y_{n+N-1}\right]\right\|+\left\|u_{n+N-1}\right\|\right)\right] \\
& +\quad \sup _{x \in\left\{x_{n}: n \in \mathbb{N}\right\}}\left\|S_{i(n+N+1)}^{p(n+N+1)} x-S_{i(n+N)}^{p(n+N)} x\right\| .
\end{aligned}
$$

By Lemma 2.5, we obtain that

$$
\lim _{n \rightarrow \infty}\left\|x_{n+N+1}-x_{n+N}\right\|=0 .
$$


Furthermore,

$$
\left\|x_{n+N}-x_{n}\right\| \leq\left\|x_{n+N}-x_{n+N-1}\right\|+\left\|x_{n+N-1}-x_{n+N-2}\right\|+\cdots+\left\|x_{n+1}-x_{n}\right\| \longrightarrow 0, \quad \text { as } n \longrightarrow \infty \text {. }
$$

Hence,

$$
\lim _{n \rightarrow \infty}\left\|x_{n+N}-x_{n}\right\|=0
$$

Next, we show that

$$
\lim _{n \rightarrow \infty}\left\|x_{n}-u_{n}\right\|=0
$$

By the convexity of the norm $\|\cdot\|$, we have

$$
\begin{aligned}
\left\|x_{n+1}-z\right\|^{2}= & \left\|\mu_{n}\left(P_{C}\left[y_{n}\right]-z\right)+\left(1-\mu_{n}\right)\left(u_{n}-z\right)\right\|^{2} \\
\leq & \mu_{n}\left\|P_{C}\left[y_{n}\right]-z\right\|^{2}+\left(1-\mu_{n}\right)\left\|u_{n}-z\right\|^{2} \\
\leq & \mu_{n}\left\|y_{n}-z\right\|^{2}+\left(1-\mu_{n}\right)\left\|u_{n}-z\right\|^{2} \\
= & \mu_{n}\left\|\alpha_{n} \gamma f\left(x_{n}\right)+\left(I-\alpha_{n} F\right) S_{i(n+1)}^{p(n+1)} x_{n}-z\right\|^{2}+\left(1-\mu_{n}\right)\left\|u_{n}-z\right\|^{2} \\
= & \mu_{n}\left\|\alpha_{n} \gamma f\left(x_{n}\right)-\alpha_{n} F(z)+\left(I-\alpha_{n} F\right) S_{i(n+1)}^{p(n+1)} x_{n}-\left(I-\alpha_{n} F\right) z\right\|^{2}+\left(1-\mu_{n}\right)\left\|u_{n}-z\right\|^{2} \\
\leq & \mu_{n}\left\|\left(I-\alpha_{n} F\right) S_{i(n+1)}^{p(n+1)} x_{n}-\left(I-\alpha_{n} F\right) z\right\|^{2}+\mu_{n} \alpha_{n}^{2}\left\|\gamma f\left(x_{n}\right)-F(z)\right\|^{2} \\
& +2 \mu_{n} \alpha_{n}\left\langle\left(I-\alpha_{n} F\right) S_{i(n+1)}^{p(n+1)} x_{n}-\left(I-\alpha_{n} F\right) z, \gamma f\left(x_{n}\right)-F(z)\right\rangle+\left(1-\mu_{n}\right)\left\|u_{n}-z\right\|^{2} \\
\leq & \mu_{n}\left(1-\alpha_{n} \bar{\gamma}\right)^{2}\left(1+h_{n+1}\right)^{2}\left\|x_{n}-z\right\|^{2}+\mu_{n} \alpha_{n}^{2}\left\|\gamma f\left(x_{n}\right)-F(z)\right\|^{2} \\
& +2 \alpha_{n} \mu_{n}\left(1-\alpha_{n} \bar{\gamma}\right)\left\|\gamma f\left(x_{n}\right)-F(z)\right\|\left\|x_{n}-z\right\|+\left(1-\mu_{n}\right)\left\|u_{n}-z\right\|^{2} \\
\leq & \mu_{n}\left(1-\alpha_{n} \bar{\gamma}\right)\left(1+2 h_{n+1}+h_{n+1}^{2}\right)\left\|x_{n}-z\right\|^{2}+\mu_{n} \alpha_{n}^{2}\left\|\gamma f\left(x_{n}\right)-F(z)\right\|^{2} \\
& +2 \alpha_{n} \mu_{n}\left(1-\alpha_{n} \bar{\gamma}\right)\left\|\gamma f\left(x_{n}\right)-F(z)\right\|\left\|x_{n}-z\right\|+\left(1-\mu_{n}\right)\left\|u_{n}-z\right\|^{2} \\
= & \mu_{n}\left(1-\alpha_{n} \bar{\gamma}\right)\left(1+h_{n+1}^{*}\right)\left\|x_{n}-z\right\|^{2}+\mu_{n} \alpha_{n}^{2}\left\|\gamma f\left(x_{n}\right)-F(z)\right\|^{2} \\
& +2 \alpha_{n} \mu_{n}\left(1-\alpha_{n} \bar{\gamma}\right)\left\|\gamma f\left(x_{n}\right)-F(z)\right\|\left\|x_{n}-z\right\|+\left(1-\mu_{n}\right)\left\|u_{n}-z\right\|^{2},
\end{aligned}
$$


where $h_{n+1}^{*}=2 h_{n+1}+h_{n+1}^{2}$. From Lemma 2.3, we get

$$
\begin{aligned}
\left\|u_{n}-z\right\|^{2} & =\left\|T_{r}\left(x_{n}-r A x_{n}\right)-T_{r}(z-r A z)\right\|^{2} \\
& \leq\left\|\left(x_{n}-r A x_{n}\right)-(z-r A z)\right\|^{2} \\
& \leq\left\|x_{n}-z\right\|^{2}+r(r-2 \alpha)\left\|A x_{n}-A z\right\|^{2} .
\end{aligned}
$$

Substituting (3.17) into (3.16), we have

$$
\begin{aligned}
\left\|x_{n+1}-z\right\|^{2} \leq & \mu_{n}\left(1-\alpha_{n} \bar{\gamma}\right)\left(1+h_{n+1}^{*}\right)\left\|x_{n}-z\right\|^{2}+\mu_{n} \alpha_{n}^{2}\left\|\gamma f\left(x_{n}\right)-F(z)\right\|^{2} \\
& +2 \alpha_{n} \mu_{n}\left(1-\alpha_{n} \bar{\gamma}\right)\left\|\gamma f\left(x_{n}\right)-F(z)\right\|\left\|x_{n}-z\right\| \\
& +\left(1-\mu_{n}\right)\left[\left\|x_{n}-z\right\|^{2}+r(r-2 \alpha)\left\|A x_{n}-A z\right\|^{2}\right] \\
= & \left(1-\alpha_{n} \mu_{n}\left(\bar{\gamma}-\frac{h_{n+1}^{*}}{\alpha_{n}}\right)\right)\left\|x_{n}-z\right\|^{2}-\alpha_{n} \mu_{n} h_{n+1} \bar{\gamma}\left\|x_{n}-z\right\|^{2} \\
& +\mu_{n} \alpha_{n}^{2}\left\|\gamma f\left(x_{n}\right)-F(z)\right\|^{2}+2 \alpha_{n} \mu_{n}\left(1-\alpha_{n} \bar{\gamma}\right)\left\|\gamma f\left(x_{n}\right)-F(z)\right\|\left\|x_{n}-z\right\| \\
& +\left(1-\mu_{n}\right) r(r-2 \alpha)\left\|A x_{n}-A z\right\|^{2} .
\end{aligned}
$$

Therefore,

$$
\begin{aligned}
\left(1-\mu_{n}\right) r(2 \alpha-r)\left\|A x_{n}-A z\right\|^{2} \leq & \left(1-\alpha_{n} \mu_{n}\left(\bar{\gamma}-\frac{h_{n+1}^{*}}{\alpha_{n}}\right)\right)\left\|x_{n}-z\right\|^{2}-\left\|x_{n+1}-z\right\|^{2} \\
& +\mu_{n} \alpha_{n}^{2}\left\|\gamma f\left(x_{n}\right)-F(z)\right\|^{2}+2 \alpha_{n} \mu_{n}\left(1-\alpha_{n} \bar{\gamma}\right) \\
& \times\left\|\gamma f\left(x_{n}\right)-F(z)\right\|\left\|x_{n}-z\right\| \\
\leq & \left\|x_{n}-z\right\|^{2}-\left\|x_{n+1}-z\right\|^{2}+\mu_{n} \alpha_{n}^{2}\left\|\gamma f\left(x_{n}\right)-F(z)\right\|^{2} \\
& +2 \alpha_{n} \mu_{n}\left(1-\alpha_{n} \bar{\gamma}\right)\left\|\gamma f\left(x_{n}\right)-F(z)\right\|\left\|x_{n}-z\right\| \\
\leq & \left(\left\|x_{n}-z\right\|+\left\|x_{n+1}-z\right\|\right)\left\|x_{n}-x_{n+1}\right\| \\
& +\mu_{n} \alpha_{n}^{2}\left\|\gamma f\left(x_{n}\right)-F(z)\right\|^{2} \\
& +2 \alpha_{n} \mu_{n}\left(1-\alpha_{n} \bar{\gamma}\right)\left\|\gamma f\left(x_{n}\right)-F(z)\right\|\left\|x_{n}-z\right\| .
\end{aligned}
$$

Since $\liminf _{n \rightarrow \infty}\left(1-\mu_{n}\right) r(2 \alpha-r)>0,\left\|x_{n}-x_{n+1}\right\| \rightarrow 0$ and $\alpha_{n} \rightarrow 0$, we derive

$$
\lim _{n \rightarrow \infty}\left\|A x_{n}-A z\right\|=0 \text {. }
$$


From Lemma 2.2, we obtain

$$
\begin{aligned}
\left\|u_{n}-z\right\|^{2}= & \left\|T_{r}\left(x_{n}-r A x_{n}\right)-T_{r}(z-r A z)\right\|^{2} \\
\leq & \left\langle\left(x_{n}-r A x_{n}\right)-(z-r A z), u_{n}-z\right\rangle \\
= & \frac{1}{2}\left(\left\|\left(x_{n}-r A x_{n}\right)-(z-r A z)\right\|^{2}+\left\|u_{n}-z\right\|^{2}\right. \\
& \left.\quad-\left\|\left(x_{n}-z\right)-r\left(A x_{n}-A z\right)-\left(u_{n}-z\right)\right\|^{2}\right) \\
\leq & \frac{1}{2}\left(\left\|x_{n}-z\right\|^{2}+\left\|u_{n}-z\right\|^{2}-\left\|\left(x_{n}-u_{n}\right)-r\left(A x_{n}-A z\right)\right\|^{2}\right) \\
= & \frac{1}{2}\left(\left\|x_{n}-z\right\|^{2}+\left\|u_{n}-z\right\|^{2}-\left\|x_{n}-u_{n}\right\|^{2}+2 r\left\langle x_{n}-u_{n}, A x_{n}-A z\right\rangle-r^{2}\left\|A x_{n}-A z\right\|^{2}\right) .
\end{aligned}
$$

Thus, we deduce

$$
\left\|u_{n}-z\right\|^{2} \leq\left\|x_{n}-z\right\|^{2}-\left\|x_{n}-u_{n}\right\|^{2}+2 r\left\|x_{n}-u_{n}\right\|\left\|A x_{n}-A z\right\| .
$$

By (3.16) and (3.22), we have

$$
\begin{aligned}
\left\|x_{n+1}-z\right\|^{2} \leq & \mu_{n}\left(1-\alpha_{n} \bar{\gamma}\right)\left(1+h_{n+1}^{*}\right)\left\|x_{n}-z\right\|^{2}+\mu_{n} \alpha_{n}^{2}\left\|\gamma f\left(x_{n}\right)-F(z)\right\|^{2} \\
& +2 \alpha_{n} \mu_{n}\left(1-\alpha_{n} \bar{\gamma}\right)\left\|\gamma f\left(x_{n}\right)-F(z)\right\|\left\|x_{n}-z\right\| \\
& +\left(1-\mu_{n}\right)\left[\left\|x_{n}-z\right\|^{2}-\left\|x_{n}-u_{n}\right\|^{2}+2 r\left\|x_{n}-u_{n}\right\|\left\|A x_{n}-A z\right\|\right] \\
\leq & \left(1-\alpha_{n} \mu_{n}\left(\bar{\gamma}-\frac{h_{n+1}^{*}}{\alpha_{n}}\right)\right)\left\|x_{n}-z\right\|^{2}+\mu_{n} \alpha_{n}^{2}\left\|\gamma f\left(x_{n}\right)-F(z)\right\|^{2} \\
& +2 \alpha_{n} \mu_{n}\left(1-\alpha_{n} \bar{\gamma}\right)\left\|\gamma f\left(x_{n}\right)-F(z)\right\|\left\|x_{n}-z\right\| \\
& +\left(1-\mu_{n}\right)\left[-\left\|x_{n}-u_{n}\right\|^{2}+2 r\left\|x_{n}-u_{n}\right\|\left\|A x_{n}-A z\right\|\right] .
\end{aligned}
$$

Therefore,

$$
\begin{aligned}
\left(1-\mu_{n}\right)\left\|x_{n}-u_{n}\right\|^{2} \leq & \left\|x_{n}-z\right\|^{2}-\left\|x_{n+1}-z\right\|^{2}+\mu_{n} \alpha_{n}^{2}\left\|\gamma f\left(x_{n}\right)-F(z)\right\|^{2} \\
& +2 \alpha_{n} \mu_{n}\left(1-\alpha_{n} \bar{\gamma}\right)\left\|\gamma f\left(x_{n}\right)-F(z)\right\|\left\|x_{n}-z\right\| \\
& +\left(1-\mu_{n}\right)\left[2 r\left\|x_{n}-u_{n}\right\|\left\|A x_{n}-A z\right\|\right] \\
\leq & \left(\left\|x_{n}-z\right\|-\left\|x_{n+1}-z\right\|\right)\left\|x_{n}-x_{n+1}\right\|+\mu_{n} \alpha_{n}^{2}\left\|\gamma f\left(x_{n}\right)-F(z)\right\|^{2} \\
& +2 \alpha_{n} \mu_{n}\left(1-\alpha_{n} \bar{\gamma}\right)\left\|\gamma f\left(x_{n}\right)-F(z)\right\|\left\|x_{n}-z\right\| \\
& +2 r\left(1-\mu_{n}\right)\left\|x_{n}-u_{n}\right\|\left\|A x_{n}-A z\right\| .
\end{aligned}
$$


Since $\liminf _{n \rightarrow \infty}\left(1-\mu_{n}\right)>0, \alpha_{n} \rightarrow 0,\left\|x_{n+1}-x_{n}\right\| \rightarrow 0$ and $\left\|A x_{n}-A z\right\| \rightarrow 0$, we derive that

$$
\lim _{n \rightarrow \infty}\left\|x_{n}-u_{n}\right\|=0
$$

Next, we show that

$$
\lim _{n \rightarrow \infty}\left\|x_{n}-S_{i(n+N)} S_{i(n+N-1)} S_{i(n+N-2)} \cdots S_{i(n+2)} S_{i(n+1)} x_{n}\right\|=0 .
$$

By using (3.14), it suffices to show that

$$
\lim _{n \rightarrow \infty}\left\|x_{n+N}-S_{i(n+N)} S_{i(n+N-1)} S_{i(n+N-2)} \cdots S_{i(n+2)} S_{i(n+1)} x_{n}\right\|=0 .
$$

Observe that

$$
\begin{aligned}
\left\|x_{n+N-1}-S_{i(n+N)}^{p(n+N)} x_{n+N-1}\right\| \leq & \left\|x_{n+N}-x_{n+N-1}\right\|+\left\|x_{n+N}-S_{i(n+N)}^{p(n+N)} x_{n+N-1}\right\| \\
\leq & \left\|x_{n+N}-x_{n+N-1}\right\|+\mu_{n+N-1}\left\|P_{C}\left[y_{n+N-1}\right]-S_{i(n+N)}^{p(n+N)} x_{n+N-1}\right\| \\
& +\left(1-\mu_{n+N-1}\right)\left\|u_{n+N-1}-S_{i(n+N)}^{p(n+N)} x_{n+N-1}\right\| \\
\leq & \left\|x_{n+N}-x_{n+N-1}\right\|+\mu_{n+N-1}\left\|y_{n+N-1}-S_{i(n+N)}^{p(n+N)} x_{n+N-1}\right\| \\
& +\left(1-\mu_{n+N-1}\right)\left\|u_{n+N-1}-S_{i(n+N)}^{p(n+N)} x_{n+N-1}\right\| \\
\leq & \left\|x_{n+N}-x_{n+N-1}\right\|+\mu_{n+N-1} \alpha_{n+N-1} \\
& \times\left(\left\|r f\left(x_{n+N-1}\right)\right\|+\left\|F S_{i(n+N)}^{p(n+N)} x_{n+N-1}\right\|\right) \\
& +\left(1-\mu_{n+N-1}\right)\left\|u_{n+N-1}-x_{n+N-1}\right\| \\
& +\left(1-\mu_{n+N-1}\right)\left\|x_{n+N-1}-S_{i(n+N)}^{p(n+N)} x_{n+N-1}\right\| .
\end{aligned}
$$

Hence,

$$
\begin{aligned}
\left\|x_{n+N-1}-S_{i(n+N)}^{p(n+N)} x_{n+N-1}\right\| \leq & \frac{1}{\mu_{n+N-1}}\left\|x_{n+N}-x_{n+N-1}\right\| \\
& +\alpha_{n+N-1}\left(\left\|\gamma f\left(x_{n+N-1}\right)\right\|+\left\|F S_{i(n+N)}^{p(n+N)} x_{n+N-1}\right\|\right) \\
& +\frac{\left(1-\mu_{n+N-1}\right)}{\mu_{n+N-1}}\left\|u_{n+N-1}-x_{n+N-1}\right\| .
\end{aligned}
$$

From (3.14), (3.25), $\lim _{n \rightarrow \infty} \alpha_{n}=0$, and (C2), we have

$$
\left\|x_{n+N-1}-S_{i(n+N)}^{p(n+N)} x_{n+N-1}\right\| \longrightarrow 0 \quad \text { as } n \longrightarrow \infty .
$$


Since $S_{i(n)}$ is Lipschitz with constant $L_{i(n)}$ for each $i(n) \in\{1,2, \ldots, N\}$ and for $L=$ $\max _{1 \leq i \leq N}\left\{L_{i(n)}\right\}$, and for any positive number $n \geq 1, n=(p(n)-1) N+i(n)$, we have

$$
\begin{aligned}
\left\|x_{n+N-1}-S_{i(n+N)} x_{n+N-1}\right\| \leq & \left\|x_{n+N-1}-S_{i(n+N)}^{p(n+N)} x_{n+N-1}\right\|+\left\|S_{i(n+N)}^{p(n+N)} x_{n+N-1}-S_{i(n+N)} x_{n+N-1}\right\| \\
\leq & \left\|x_{n+N-1}-S_{i(n+N)}^{p(n+N)} x_{n+N-1}\right\|+L\left\|S_{i(n+N)}^{p(n+N)-1} x_{n+N-1}-x_{n+N-1}\right\| \\
\leq & \left\|x_{n+N-1}-S_{i(n+N)}^{p(n+N)} x_{n+N-1}\right\| \\
& +L\left(\left\|S_{i(n+N)-1}^{p(n+N)-} x_{n+N-1}-S_{i(n)}^{p(n+N)-1} x_{n-1}\right\|\right. \\
& \left.+\left\|S_{i(n)}^{p(n+N)-1} x_{n-1}-x_{n-1}\right\|+\left\|x_{n-1}-x_{n+N-1}\right\|\right) .
\end{aligned}
$$

Since for each $n>N, n+N=n(\bmod N)$, and also $n=(p(n)-1) N+i(n)$, so

$$
n+N=(p(n)-1+1) N+i(n)=(p(n+N)-1) N+i(n+N)
$$

that is,

$$
p(n+N)-1=p(n), \quad i(n+N)=i(n) .
$$

Hence,

$$
\left\|S_{i(n+N)}^{p(n+N)-1} x_{n+N-1}-S_{i(n)}^{p(n+N)-1} x_{n-1}\right\|=\left\|S_{i(n)}^{p(n)} x_{n+N-1}-S_{i(n)}^{p(n)} x_{n-1}\right\| \leq L\left\|x_{n+N-1}-x_{n-1}\right\| .
$$

Also,

$$
\left\|S_{i(n)}^{p(n+N)-1} x_{n-1}-x_{n-1}\right\|=\left\|S_{i(n)}^{p(n)} x_{n-1}-x_{n-1}\right\| .
$$

Therefore, substituting (3.34) and (3.35) into (3.31), we have

$$
\begin{aligned}
\left\|x_{n+N-1}-S_{i(n+N)} x_{n+N-1}\right\| \leq & \left\|x_{n+N-1}-S_{i(n+N)}^{p(n+N)} x_{n+N-1}\right\|+L^{2}\left\|x_{n+N-1}-x_{n-1}\right\| \\
& +L\left\|S_{i(n)}^{p(n)} x_{n-1}-x_{n-1}\right\|+L\left\|x_{n-1}-x_{n+N-1}\right\| .
\end{aligned}
$$

From (3.30) and (3.14), we have

$$
\lim _{n \rightarrow \infty}\left\|x_{n+N-1}-S_{i(n+N)} x_{n+N-1}\right\|=0 .
$$

Also,

$$
\left\|x_{n+N}-S_{i(n+N)} x_{n+N-1}\right\| \leq\left\|x_{n+N}-x_{n+N-1}\right\|+\left\|x_{n+N-1}-S_{i(n+N)} x_{n+N-1}\right\|,
$$


so that

$$
x_{n+N-1}-S_{i(n+N)} x_{n+N-1} \longrightarrow 0 \text { as } n \longrightarrow \infty .
$$

Indeed, noting that each $S_{i(n)}$ is Lipschitzian and using (3.39), we can calculate the following:

$$
\begin{gathered}
x_{n+N}-S_{i(n+N) x_{n+N-1}} \longrightarrow 0 \text { as } n \longrightarrow \infty, \\
S_{i(n+N)} x_{n+N-1}-S_{i(n+N)} S_{i(n+N-1)} x_{n+N-2} \quad \text { as } n \longrightarrow \infty, \\
\vdots \\
S_{i(n+N)} S_{i(n+N-1)} \cdots S_{i(n+2)} x_{n+1}-S_{i(n+N)} S_{i(n+N-1)} \cdots S_{i(n+2)} S_{i(n+1)} x_{n} \longrightarrow 0 \quad \text { as } n \longrightarrow \infty .
\end{gathered}
$$

It follows from (3.40) that

$$
x_{n+N}-S_{i(n+N)} S_{i(n+N-1)} \cdots S_{i(n+1)} x_{n} \longrightarrow 0 \quad \text { as } n \longrightarrow \infty .
$$

Using (3.14), we have

$$
x_{n}-S_{i(n+N)} S_{i(n+N-1)} \cdots S_{i(n+1)} x_{n} \longrightarrow 0 \quad \text { as } n \longrightarrow \infty .
$$

Hence (3.26) is proved. Let $\Phi=P_{\Omega}$. Then, $\Phi(I-F-\gamma f)$ is a contraction on $C$. In fact, from Lemma 2.7(i), we have

$$
\begin{aligned}
\|\Phi(I-F-\gamma f) x-\Phi(I-F-\gamma f) y\| & \leq\|(I-F-\gamma f) x-(I-F-\gamma f) y\| \\
& \leq\|(I-F) x-(I-F) y\|+\gamma\|f(x)-f(y)\| \\
& \leq \sqrt{\frac{1-\delta}{\lambda}}\|x-y\|+\alpha \gamma\|x-y\| \\
& =\left(\sqrt{\frac{1-\delta}{\imath}}+\alpha \gamma\right)\|x-y\|, \quad \forall x, y \in C .
\end{aligned}
$$

Therefore, $\Phi(I-F-\gamma f)$ is a contraction on $C$ with coefficient $(\sqrt{(1-\delta) / \lambda}+\alpha \gamma) \in(0,1)$. Thus, by Banach contraction principal, $P_{\Omega}(I-F-\gamma f)$ has a uninique fixed point $x^{*}$, that is $P_{\Omega}(I-F-\gamma f) x^{*}=x^{*}$ which mean that $x^{*}$ is the unique solution in $\Omega$ of the variational inequality (3.2). Next, we show that

$$
\limsup _{n \rightarrow \infty}\left\langle\gamma f\left(x^{*}\right)-F x^{*}, x_{n}-x^{*}\right\rangle \leq 0
$$


Let $\left\{x_{n_{j}}\right\}$ be a subsequence of $\left\{x_{n}\right\}$ such that

$$
\limsup _{n \rightarrow \infty}\left\langle\gamma f\left(x^{*}\right)-F x^{*}, x_{n}-x^{*}\right\rangle=\lim _{j \rightarrow \infty}\left\langle\gamma f\left(x^{*}\right)-F x^{*}, x_{n_{j}}-x^{*}\right\rangle .
$$

Since $\left\{x_{n}\right\}$ is bounded, we may also assume that there exists some $\tilde{x} \in H$ such that $x_{n_{j}}-\tilde{x}$. Since the family $\left\{S_{i}\right\}_{i=1}^{N}$ is finite, passing to a further subsequence if necessary, we may further assume, for some $i(n) \in\{1,2, \ldots, N\}$, it follows that

$$
x_{n_{j}}-S_{i(n+N)} S_{i(n+N-1)} \cdots S_{i(n+1)} x_{n_{j}} \longrightarrow 0 \quad \text { as } j \longrightarrow \infty .
$$

By Lemma 2.4, we obtain

$$
\tilde{x} \in F\left(S_{i(n+N)} S_{i(n+N-1)} \cdots S_{i(n+1)}\right),
$$

so this implies that $\tilde{x} \in \Gamma$. Next, we show $\tilde{x} \in$ EP. Since $u_{n}=T_{r}\left(x_{n}-r A x_{n}\right)$, for any $y \in C$, we have

$$
\phi\left(u_{n}, y\right)+\frac{1}{r}\left\langle y-u_{n}, u_{n}-\left(x_{n}-r A x_{n}\right)\right\rangle \geq 0
$$

From the monotonicity of $F$, we have

$$
\frac{1}{r}\left\langle y-u_{n}, u_{n}-\left(x_{n}-r A x_{n}\right)\right\rangle \geq \phi\left(y, u_{n}\right), \quad \forall y \in C .
$$

Hence,

$$
\left\langle y-u_{n}, \frac{u_{n_{i}}-x_{n_{i}}}{r}+A x_{n_{i}}\right\rangle \geq \phi\left(y, u_{n_{i}}\right), \quad \forall y \in C .
$$

Put $z_{t}=t y+(1-t) \tilde{x}$ for all $t \in(0,1]$ and $y \in C$. Then, we have $z_{t} \in C$. So, from (3.50), we have

$$
\begin{aligned}
\left\langle z_{t}-u_{n_{i}}, A z_{t}\right\rangle \geq & \left\langle z_{t}-u_{n_{i}}, A z_{t}\right\rangle-\left\langle z_{t}-u_{n_{i}}, \frac{u_{n_{i}}-x_{n_{i}}}{r}+A x_{n_{i}}\right\rangle+\phi\left(z_{t}, u_{n_{i}}\right) \\
= & \left\langle z_{t}-u_{n_{i}}, A z_{t}-A u_{n_{i}}\right\rangle+\left\langle z_{t}-u_{n_{i}}, A u_{n_{i}}-A x_{n_{i}}\right\rangle \\
& +\left\langle z_{t}-u_{n_{i}}, \frac{u_{n_{i}}-x_{n_{i}}}{r}\right\rangle+\phi\left(z_{t}, u_{n_{i}}\right) .
\end{aligned}
$$

Note that $\left\|A u_{n_{i}}-A x_{n_{i}}\right\| \leq(1 / \alpha)\left\|u_{n_{i}}-x_{n_{i}}\right\| \rightarrow 0$. Further, from monotonicity of $A$, we have $\left\langle z_{t}-u_{n_{i}}, \mathrm{~A} z_{t}-A u_{n_{i}}\right\rangle \geq 0$. Letting $i \rightarrow \infty$ in (3.51), we have

$$
\left\langle z_{t}-\tilde{x}, A z_{t}\right\rangle \geq \phi\left(z_{t}, \tilde{x}\right)
$$


From (A1), (A4), and (3.52), we also have

$$
\begin{aligned}
0 & =\phi\left(z_{t}, z_{t}\right) \leq t \phi\left(z_{t}, y\right)+(1-t) \phi\left(z_{t}, \tilde{x}\right) \\
& \leq t \phi\left(z_{t}, y\right)+(1-t)\left\langle z_{t}-\tilde{x}, A z_{t}\right\rangle \\
& =t \phi\left(z_{t}, y\right)+(1-t) t\left\langle y-\tilde{x}, A z_{t}\right\rangle
\end{aligned}
$$

and, hence,

$$
0 \leq \phi\left(z_{t}, y\right)+(1-t)\left\langle A z_{t}, y-\tilde{x}\right\rangle
$$

Letting $t \rightarrow 0$ in (3.54) and using (A3), we have, for each $y \in C$,

$$
0 \leq \phi(\tilde{x}, y)+\langle y-\tilde{x}, A \tilde{x}\rangle
$$

This implies that $\tilde{x} \in \mathrm{EP}$. Therefore, $\tilde{x} \in \Omega$. Therefore,

$$
\limsup _{n \rightarrow \infty}\left\langle\gamma f\left(x^{*}\right)-F x^{*}, x_{n}-x^{*}\right\rangle=\left\langle\gamma f\left(x^{*}\right)-F x^{*}, \tilde{x}-x^{*}\right\rangle \leq 0 .
$$

Finally, we prove that $x_{n} \rightarrow x^{*}$ as $n \rightarrow \infty$. From Lemma 2.7 and (1.23), we obtain

$$
\begin{aligned}
\left\|x_{n+1}-x^{*}\right\|^{2}= & \left\|\mu_{n}\left(P_{C}\left[y_{n}\right]-x^{*}\right)+\left(1-\mu_{n}\right)\left(u_{n}-x^{*}\right)\right\|^{2} \\
\leq & \mu_{n}\left\|P_{C}\left[y_{n}\right]-x^{*}\right\|^{2}+\left(1-\mu_{n}\right)\left\|u_{n}-x^{*}\right\|^{2} \\
\leq & \mu_{n}\left\|y_{n}-x^{*}\right\|^{2}+\left(1-\mu_{n}\right)\left\|u_{n}-x^{*}\right\|^{2} \\
= & \mu\left\|\alpha_{n} \gamma f\left(x_{n}\right)-\alpha_{n} F\left(x^{*}\right)+\left(I-\alpha_{n} F\right) S_{i(n+1)}^{p(n+1)} x_{n}-\left(I-\alpha_{n} F\right) x^{*}\right\|^{2} \\
& +\left(1-\mu_{n}\right)\left\|u_{n}-x^{*}\right\|^{2} \\
= & \left(1-\mu_{n}\right)\left\|x_{n}-x^{*}\right\|^{2}+\mu_{n}\left\|\alpha_{n}\left(\gamma f\left(x_{n}\right)-F\left(x^{*}\right)\right)+\left(I-\alpha_{n} F\right)\left(S_{i(n+1)}^{p(n+1)} x_{n}-x^{*}\right)\right\|^{2} \\
\leq & \left(1-\mu_{n}\right)\left\|x_{n}-x^{*}\right\|^{2}+\mu_{n}\left(1-\alpha_{n} \bar{\gamma}\right)^{2}\left(1+h_{n+1}\right)^{2}\left\|x_{n}-x^{*}\right\|^{2} \\
& +2 \mu_{n} \alpha_{n}\left\langle\gamma f\left(x_{n}\right)-F\left(x^{*}\right), x_{n+1}-x^{*}\right\rangle \\
\leq & \left(1-\mu_{n}\right)\left\|x_{n}-x^{*}\right\|^{2}+\mu_{n}\left(1-\alpha_{n} \bar{\gamma}\right)\left(1+2 h_{n+1}+h_{n+1}^{2}\right)\left\|x_{n}-x^{*}\right\|^{2} \\
& +2 \mu_{n} \alpha_{n}\left\langle\gamma f\left(x_{n}\right)-F\left(x^{*}\right), x_{n+1}-x^{*}\right\rangle \\
= & \left(1-\mu_{n}\right)\left\|x_{n}-x^{*}\right\|^{2}+\mu_{n}\left(1-\alpha_{n} \bar{\gamma}\right)\left(1+h_{n+1}^{*}\right)\left\|x_{n}-x^{*}\right\|^{2} \\
& +2 \mu_{n} \alpha_{n}\left\langle\gamma f\left(x_{n}\right)-F\left(x^{*}\right), x_{n+1}-x^{*}\right\rangle
\end{aligned}
$$




$$
\begin{aligned}
& \leq\left(1-\mu_{n} \alpha_{n}\left(\bar{\gamma}-\frac{h_{n+1}^{*}}{\alpha_{n}}\right)\right)\left\|x_{n}-x^{*}\right\|^{2} \\
& \quad+2 \mu_{n} \alpha_{n}\left(\bar{\gamma}-\frac{h_{n+1}^{*}}{\alpha_{n}}\right)\left[\frac{1}{\left(\bar{\gamma}-h_{n+1}^{*} / \alpha_{n}\right)}\left\langle\gamma f\left(x_{n}\right)-F\left(x^{*}\right), x_{n+1}-x^{*}\right\rangle\right],
\end{aligned}
$$

where $h_{n+1}^{*}=2 h_{n+1}+h_{n+1}^{2}$. Hence, all conditions of Lemma 2.5 are satisfied. Therefore, $x_{n} \rightarrow$ $x^{*}$. This completes the proof.

The following example shows that there exist the sequences $\left\{\alpha_{n}\right\}$ and $\left\{\mu_{n}\right\}$ satisfying the conditions (C1) and (C2) of Theorem 3.1.

Example 3.2. For each $n \geq 0$, let $\alpha_{n}=1 /(n+1)$ and $\mu_{n}=1 / 2+1 /(n+1)$. Then, it is easy to obtain $\lim _{n \rightarrow \infty} \alpha_{n}=0, \sum_{n=0}^{\infty} \alpha_{n}=\infty$ and $\lim _{n \rightarrow \infty}\left(\alpha_{n+1} / \alpha_{n}\right)=1,0<1 / 2=\liminf _{n \rightarrow \infty} \mu_{n} \leq$ $\lim \sup _{n \rightarrow \infty} \mu_{n}=1 / 2<1$ and $\lim _{n \rightarrow \infty}\left(\left(\mu_{n+1}-\mu_{n}\right) / \alpha_{n+1}\right)=0$. Hence, conditions (C1) and (C2) of Theorem 3.1 are satisfied.

Corollary 3.3. Let $C, H, A, \phi, \Omega, f, F, r$ be as in Theorem 3.1. Let $S_{1}, S_{2}, \ldots, S_{N}: C \rightarrow C$ be a family of nonexpansive mappings. Let $T_{1}, T_{2}, \ldots, T_{N}: C \rightarrow C$ be mappings defined by (2.9). For $T_{n}:=T_{n \bmod N}$, let the sequence $\left\{x_{n}\right\}$ be generated by

$$
\begin{gathered}
\phi\left(u_{n}, y\right)+\left\langle A x_{n}, y-u_{n}\right\rangle+\frac{1}{r}\left\langle y-u_{n}, u_{n}-x_{n}\right\rangle \geq 0, \quad \forall y \in C, \\
x_{n+1}=\mu_{n} P_{C}\left[\alpha_{n} \gamma f\left(x_{n}\right)+\left(1-\alpha_{n} F\right) T_{n} x_{n}\right]+\left(1-\mu_{n}\right) u_{n}, \quad n \geq 0 .
\end{gathered}
$$

Assume that $\sum_{n=1}^{\infty} \sup _{z \in B}\left\|T_{n+1} z-T_{n} z\right\|<\infty$ for each bounded subset $B$ of $C$ and the sequences $\left\{\alpha_{n}\right\}$ and $\left\{\mu_{n}\right\}$ satisfy the following conditions:

(C1) $\lim _{n \rightarrow \infty} \alpha_{n}=0, \sum_{n=0}^{\infty} \alpha_{n}=\infty$ and $\lim _{n \rightarrow \infty}\left(\alpha_{n+1} / \alpha_{n}\right)=1$,

(C2) $0<\liminf _{n \rightarrow \infty} \mu_{n} \leq \lim \sup _{n \rightarrow \infty} \mu_{n}<1$ and $\lim _{n \rightarrow \infty}\left(\left(\mu_{n+1}-\mu_{n}\right) / \alpha_{n+1}\right)=0$.

Then the sequence $\left\{x_{n}\right\}$ converges strongly to $x^{*}$ of the following variational inequality:

$$
\left\langle(F-\gamma f) x^{*}, x-x^{*}\right\rangle \geq 0, \quad x \in \Omega,
$$

or equivalently $\tilde{x}=P_{\Omega}(I-F+\gamma f) \tilde{x}$, where $P_{\Omega}$ is the metric projection of $H$ onto $\Omega$.

Proof. By Lemma 2.6, we have

$$
\bigcap_{i=1}^{N} \operatorname{Fix}\left(T_{i}\right)=\operatorname{Fix}\left(T_{N} T_{N-1} T_{N-2} \cdots T_{1}\right)=\operatorname{Fix}\left(T_{1} T_{N} \cdots T_{2}\right)=\operatorname{Fix}\left(T_{N-1} T_{N-2} \cdots T_{1} T_{N}\right) .
$$

Therefore, the result follows from Theorem 3.1. 
Remark 3.4. As in [58, Theorem 4.1], we can generate a sequence $\left\{S_{n}\right\}$ of nonexpansive mappings satisfying the condition $\sum_{n=1}^{\infty} \sup \left\{\left\|S_{n+1} z-S_{n} z\right\|: z \in B\right\}<\infty$ for any bounded subset $B$ of $C$ by using convex combination of a general sequence $\left\{T_{k}\right\}$ of nonexpansive mappings with a common fixed point.

Setting $\gamma=1, F=I$, and $S_{n} \equiv S$, a nonexpansive mapping, in Corollary 3.3, we obtain the following result.

Corollary 3.5 ([46], Theorem 3.7). Let $C, H, A, \phi, f, r$ be as in Theorem 3.1. Let $S: C \rightarrow C$ be a nonexpansive mapping such that $\Omega:=\operatorname{EP} \cap \operatorname{Fix}(S) \neq \emptyset$. Let the sequence $\left\{x_{n}\right\}$ be generated by

$$
\begin{gathered}
\phi\left(u_{n}, y\right)+\left\langle A x_{n}, y-u_{n}\right\rangle+\frac{1}{r}\left\langle y-u_{n}, u_{n}-x_{n}\right\rangle \geq 0, \quad \forall y \in C, \\
x_{n+1}=\mu_{n} P_{C}\left[\alpha_{n} f\left(x_{n}\right)+\left(1-\alpha_{n}\right) S x_{n}\right]+\left(1-\mu_{n}\right) u_{n}, \quad n \geq 0 .
\end{gathered}
$$

Assume the sequences $\left\{\alpha_{n}\right\}$ and $\left\{\mu_{n}\right\}$ satisfy the following conditions:

(C1) $\lim _{n \rightarrow \infty} \alpha_{n}=0, \sum_{n=0}^{\infty} \alpha_{n}=\infty$ and $\lim _{n \rightarrow \infty}\left(\alpha_{n+1} / \alpha_{n}\right)=1$,

(C2) $0<\liminf _{n \rightarrow \infty} \mu_{n} \leq \lim \sup _{n \rightarrow \infty} \mu_{n}<1$ and $\lim _{n \rightarrow \infty}\left(\left(\mu_{n+1}-\mu_{n}\right) / \alpha_{n+1}\right)=0$.

Then, the sequence $\left\{x_{n}\right\}$ converges strongly to $x^{*}$ of the following variational inequality:

$$
\left\langle(I-f) x^{*}, x-x^{*}\right\rangle \geq 0, \quad x \in \Omega
$$

\section{Acknowledgments}

R. Wangkeeree is supported by The Thailand Research Fund under Grant no. MRG5380244, and U. Kamraksa is supported by grant from the program of Strategic Scholarships for Frontier Research Network for the Ph.D. Program Thai Doctoral degree from the Office of the Higher Education Commission, Thailand.

\section{References}

[1] K. Goebel and W. A. Kirk, "A fixed point theorem for asymptotically nonexpansive mappings," Proceedings of the American Mathematical Society, vol. 35, pp. 171-174, 1972.

[2] E. Blum and W. Oettli, "From optimization and variational inequalities to equilibrium problems," The Mathematics Student, vol. 63, no. 1-4, pp. 123-145, 1994.

[3] S. Takahashi and W. Takahashi, "Strong convergence theorem for a generalized equilibrium problem and a nonexpansive mapping in a Hilbert space," Nonlinear Analysis: Theory, Methods E Applications, vol. 69, no. 3, pp. 1025-1033, 2008.

[4] A. Moudafi and M. Théra, "Proximal and dynamical approaches to equilibrium problems," in IllPosed Variational Problems and Regularization Techniques (Trier, 1998), vol. 477 of Lecture Notes in Econom. and Math. Systems, pp. 187-201, Springer, Berlin, Germany, 1999.

[5] A. Moudafi, "Weak convergence theorems for nonexpansive mappings and equilibrium problems," Journal of Nonlinear and Convex Analysis, vol. 9, no. 1, pp. 37-43, 2008.

[6] P. L. Combettes and S. A. Hirstoaga, "Equilibrium programming in Hilbert spaces," Journal of Nonlinear and Convex Analysis, vol. 6, no. 1, pp. 117-136, 2005. 
[7] S. Takahashi and W. Takahashi, "Viscosity approximation methods for equilibrium problems and fixed point problems in Hilbert spaces," Journal of Mathematical Analysis and Applications, vol. 331, no. 1, pp. 506-515, 2007.

[8] L.-C. Ceng and J.-C. Yao, "A hybrid iterative scheme for mixed equilibrium problems and fixed point problems," Journal of Computational and Applied Mathematics, vol. 214, no. 1, pp. 186-201, 2008.

[9] P.-E. Maingé and A. Moudafi, "Coupling viscosity methods with the extragradient algorithm for solving equilibrium problems," Journal of Nonlinear and Convex Analysis, vol. 9, no. 2, pp. 283-294, 2008.

[10] Y. Yao, Y.-C. Liou, C. Lee, and M.-M. Wong, “Convergence theorems for equilibrium problems and fixed point problems," Fixed Point Theory, vol. 10, no. 2, pp. 347-363, 2009.

[11] M. A. Noor, Y. Yao, R. Chen, and Y.-C. Liou, "An iterative method for fixed point problems and variational inequality problems," Mathematical Communications, vol. 12, no. 1, pp. 121-132, 2007.

[12] R. Wangkeeree, "An extragradient approximation method for equilibrium problems and fixed point problems of a countable family of nonexpansive mappings," Fixed Point Theory and Applications, vol. 2008, Article ID 134148, 17 pages, 2008.

[13] R. Wangkeeree and U. Kamraksa, "An iterative approximation method for solving a general system of variational inequality problems and mixed equilibrium problems," Nonlinear Analysis: Hybrid Systems, vol. 3, no. 4, pp. 615-630, 2009.

[14] L.-C. Ceng, S. Al-Homidan, Q. H. Ansari, and J.-C. Yao, "An iterative scheme for equilibrium problems and fixed point problems of strict pseudo-contraction mappings," Journal of Computational and Applied Mathematics, vol. 223, no. 2, pp. 967-974, 2009.

[15] L. C. Ceng, S. Schaible, and J. C. Yao, "Implicit iteration scheme with perturbed mapping for equilibrium problems and fixed point problems of finitely many nonexpansive mappings," Journal of Optimization Theory and Applications, vol. 139, no. 2, pp. 403-418, 2008.

[16] L.-C. Ceng and J.-C. Yao, "A relaxed extragradient-like method for a generalized mixed equilibrium problem, a general system of generalized equilibria and a fixed point problem," Nonlinear Analysis: Theory, Methods \& Applications, vol. 72, no. 3-4, pp. 1922-1937, 2010.

[17] S.-S. Chang, H. W. Joseph Lee, and C. K. Chan, "A new method for solving equilibrium problem fixed point problem and variational inequality problem with application to optimization," Nonlinear Analysis: Theory, Methods E Applications, vol. 70, no. 9, pp. 3307-3319, 2009.

[18] W. Chantarangsi, C. Jaiboon, and P. Kumam, "A viscosity hybrid steepest descent method for generalized mixed equilibrium problems and variational inequalities for relaxed cocoercive mapping in Hilbert spaces," Abstract and Applied Analysis, vol. 2010, Article ID 390972, 39 pages, 2010.

[19] F. Cianciaruso, G. Marino, L. Muglia, and Y. Yao, "A hybrid projection algorithm for finding solutions of mixed equilibrium problem and variational inequality problem," Fixed Point Theory and Applications, vol. 2010, Article ID 383740, 19 pages, 2010.

[20] F. Cianciaruso, G. Marino, L. Muglia, and Y. Yao, “On a two-step algorithm for hierarchical fixed point problems and variational inequalities," Journal of Inequalities and Applications, vol. 2009, Article ID 208692, 13 pages, 2009.

[21] V. Colao, G. L. Acedo, and G. Marino, "An implicit method for finding common solutions of variational inequalities and systems of equilibrium problems and fixed points of infinite family of nonexpansive mappings," Nonlinear Analysis: Theory, Methods E Applications, vol. 71, no. 7-8, pp. 27082715, 2009.

[22] V. Colao, G. Marino, and H.-K. Xu, "An iterative method for finding common solutions of equilibrium and fixed point problems," Journal of Mathematical Analysis and Applications, vol. 344, no. 1, pp. 340352, 2008.

[23] P. L. Combettes, "Strong convergence of block-iterative outer approximation methods for convex optimization," SIAM Journal on Control and Optimization, vol. 38, no. 2, pp. 538-565, 2000.

[24] P. L. Combettes and J.-C. Pesquet, "Proximal thresholding algorithm for minimization over orthonormal bases," SIAM Journal on Optimization, vol. 18, no. 4, pp. 1351-1376, 2007.

[25] F. Deutsch and I. Yamada, "Minimizing certain convex functions over the intersection of the fixed point sets of nonexpansive mappings," Numerical Functional Analysis and Optimization, vol. 19, no. 1-2, pp. 33-56, 1998.

[26] J. S. Jung, "Strong convergence of composite iterative methods for equilibrium problems and fixed point problems," Applied Mathematics and Computation, vol. 213, no. 2, pp. 498-505, 2009. 
[27] C. Klineam, S. Suantai, and W. Takahashi, "Strong convergence of generalized projection algorithms for nonlinear operators," Abstract and Applied Analysis, vol. 2009, Article ID 649831, 18 pages, 2009.

[28] G. Marino and H.-K. Xu, "A general iterative method for nonexpansive mappings in Hilbert spaces," Journal of Mathematical Analysis and Applications, vol. 318, no. 1, pp. 43-52, 2006.

[29] A. Moudafi, "Viscosity approximation methods for fixed-points problems," Journal of Mathematical Analysis and Applications, vol. 241, no. 1, pp. 46-55, 2000.

[30] N. Nadezhkina and W. Takahashi, "Weak convergence theorem by an extragradient method for nonexpansive mappings and monotone mappings," Journal of Optimization Theory and Applications, vol. 128, no. 1, pp. 191-201, 2006.

[31] Z. Opial, "Weak convergence of the sequence of successive approximations for nonexpansive mappings," Bulletin of the American Mathematical Society, vol. 73, pp. 591-597, 1967.

[32] J.-W. Peng, S.-Y. Wu, and J.-C. Yao, "A new iterative method for finding common solutions of a system of equilibrium problems, fixed-point problems, and variational inequalities," Abstract and Applied Analysis, vol. 2010, Article ID 428293, 27 pages, 2010.

[33] J.-W. Peng and J.-C. Yao, "A new hybrid-extragradient method for generalized mixed equilibrium problems, fixed point problems and variational inequality problems," Taiwanese Journal of Mathematics, vol. 12, no. 6, pp. 1401-1432, 2008.

[34] J.-W. Peng and J.-C. Yao, "Ishikawa iterative algorithms for a generalized equilibrium problem and fixed point problems of a pseudo-contraction mapping," Journal of Global Optimization, vol. 46, no. 3, pp. 331-345, 2010.

[35] H. Piri and H. Vaezi, "Strong convergence of a generalized iterative method for semigroups of nonexpansive mappings in Hilbert spaces," Fixed Point Theory and Applications, vol. 2010, Article ID 907275, 16 pages, 2010.

[36] S. Plubtieng and R. Punpaeng, "A new iterative method for equilibrium problems and fixed point problems of nonexpansive mappings and monotone mappings," Applied Mathematics and Computation, vol. 197, no. 2, pp. 548-558, 2008.

[37] X. Qin, Y. J. Cho, and S. M. Kang, "Convergence theorems of common elements for equilibrium problems and fixed point problems in Banach spaces," Journal of Computational and Applied Mathematics, vol. 225, no. 1, pp. 20-30, 2009.

[38] S. Saewan, P. Kumam, and K. Wattanawitoon, "Convergence theorem based on a new hybrid projection method for finding a common solution of generalized equilibrium and variational inequality problems in Banach spaces," Abstract and Applied Analysis, vol. 2010, Article ID 734126, 25 pages, 2010.

[39] W. Takahashi, Nonlinear Functional Analysis, Fixed Point Theory and Its Applications, Yokohama Publishers, Yokohama, Japan, 2000.

[40] W. Takahashi and M. Toyoda, "Weak convergence theorems for nonexpansive mappings and monotone mappings," Journal of Optimization Theory and Applications, vol. 118, no. 2, pp. 417-428, 2003.

[41] W. Takahashi, T. Tamura, and M. Toyoda, "Approximation of common fixed points of a family of finite nonexpansive mappings in Banach spaces," Scientiae Mathematicae Japonicae, vol. 56, no. 3, pp. 475-480, 2002.

[42] S. Wang, G. Marino, and F. Wang, "Strong convergence theorems for a generalized equilibrium problem with a relaxed monotone mapping and a countable family of nonexpansive mappings in a Hilbert space," Fixed Point Theory and Applications, vol. 2010, Article ID 230304, 22 pages, 2010.

[43] H. K. Xu, "An iterative approach to quadratic optimization," Journal of Optimization Theory and Applications, vol. 116, no. 3, pp. 659-678, 2003.

[44] H.-K. Xu, "Iterative algorithms for nonlinear operators," Journal of the London Mathematical Society, vol. 66, no. 1, pp. 240-256, 2002.

[45] H. K. Xu, "Existence and convergence for fixed points of mappings of asymptotically nonexpansive type," Nonlinear Analysis: Theory, Methods \& Applications, vol. 16, no. 12, pp. 1139-1146, 1991.

[46] Y. Yao and Y.-C. Liou, "Composite algorithms for minimization over the solutions of equilibrium problems and fixed point problems," Abstract and Applied Analysis, vol. 2010, Article ID 763506, 19 pages, 2010.

[47] Y. Yao, Y. C. Liou, and G. Marino, "Strong convergence of two iterative algorithms for nonexpansive mappings in Hilbert spaces," Fixed Point Theory and Applications, vol. 2009, Article ID 279058, 7 pages, 2009.

[48] Y. Yao, Y.-C. Liou, and Y.-J. Wu, "An extragradient method for mixed equilibrium problems and fixed point problems," Fixed Point Theory and Applications, vol. 2009, Article ID 632819, 15 pages, 2009. 
[49] Y. Yao, Y. C. Liou, and J. C. Yao, "A new hybrid iterative algorithm for fixed point problems, variational inequality problems, and mixed equilibrium problems," Fixed Point Theory and Applications, vol. 2008, Article ID 417089, 15 pages, 2008.

[50] Y. Yao, Y.-C. Liou, and J.-C. Yao, "An extragradient method for fixed point problems and variational inequality problems," Journal of Inequalities and Applications, vol. 2007, Article ID 38752, 12 pages, 2007.

[51] Y. Yao, Y.-C. Liou, and J.-C. Yao, "An iterative algorithm for approximating convex minimization problem," Applied Mathematics and Computation, vol. 188, no. 1, pp. 648-656, 2007.

[52] Y. Yao, Y. C. Liou, and J. C. Yao, "Convergence theorem for equilibrium problems and fixed point problems of infinite family of nonexpansive mappings," Fixed Point Theory and Applications, vol. 2007, Article ID 64363, 12 pages, 2007.

[53] Y. Yao, M. A. Noor, R. Chen, and Y.-C. Liou, "Strong convergence of three-step relaxed hybrid steepest-descent methods for variational inequalities," Applied Mathematics and Computation, vol. 201, no. 1-2, pp. 175-183, 2008.

[54] Y. Yao and J.-C. Yao, “On modified iterative method for nonexpansive mappings and monotone mappings," Applied Mathematics and Computation, vol. 186, no. 2, pp. 1551-1558, 2007.

[55] L.-C. Zeng, Q. H. Ansari, D. S. Shyu, and J.-C. Yao, "Strong and weak convergence theorems for common solutions of generalized equilibrium problems and zeros of maximal monotone operators," Fixed Point Theory and Applications, vol. 2010, Article ID 590278, 33 pages, 2010.

[56] L.-C. Ceng, S. Kum, and J.-C. Yao, "Algorithm for solving a generalized mixed equilibrium problem with perturbation in a Banach space," Fixed Point Theory and Applications, vol. 2010, Article ID 794503, 22 pages, 2010.

[57] S.-S. Zhang, H.-W. Lee, and C.-K. Chan, “Quadratic minimization for equilibrium problem variational inclusion and fixed point problem," Applied Mathematics and Mechanics, vol. 31, no. 7, pp. 917-928, 2010.

[58] K. Aoyama, Y. Kimura, W. Takahashi, and M. Toyoda, "Approximation of common fixed points of a countable family of nonexpansive mappings in a Banach space," Nonlinear Analysis: Theory, Methods E Applications, vol. 67, no. 8, pp. 2350-2360, 2007. 


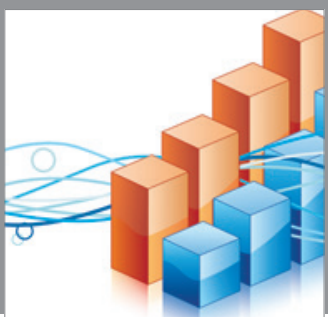

Advances in

Operations Research

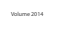

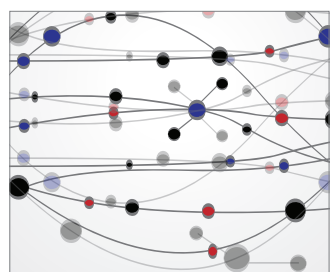

\section{The Scientific} World Journal
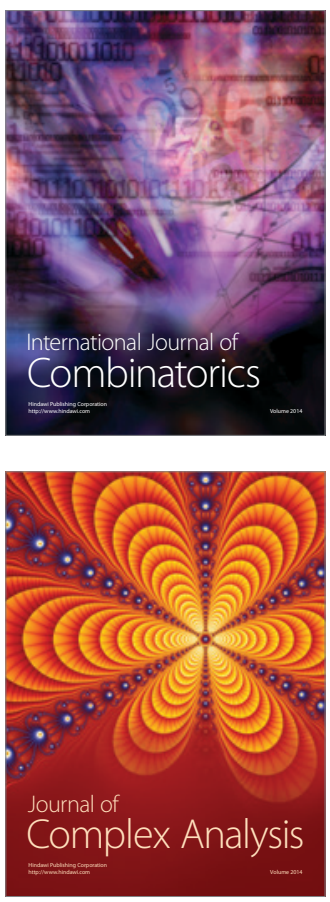

International Journal of

Mathematics and

Mathematical

Sciences
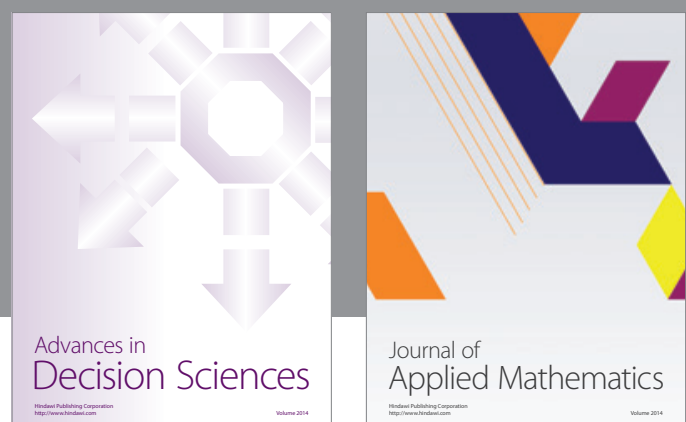

Journal of

Applied Mathematics
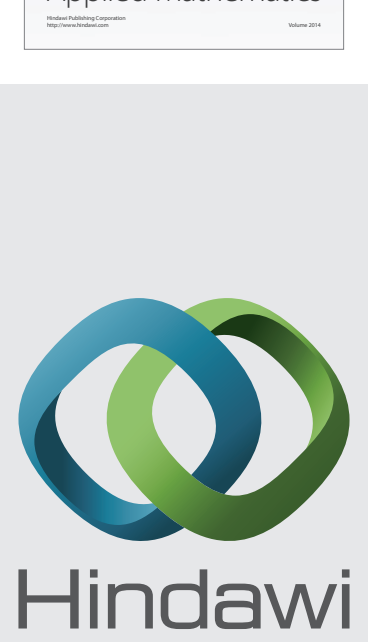

Submit your manuscripts at http://www.hindawi.com
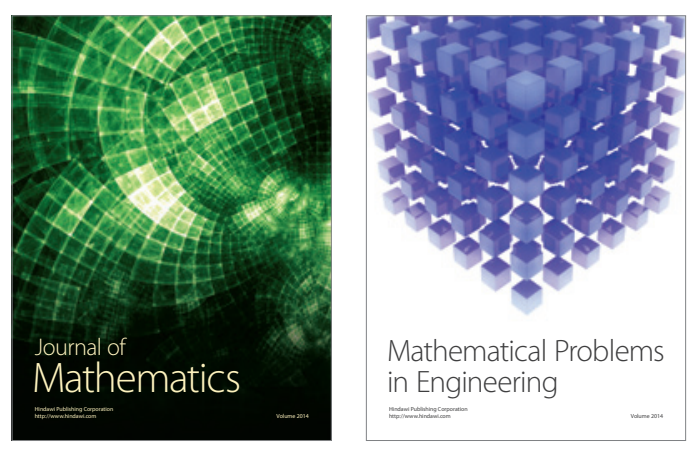

Mathematical Problems in Engineering
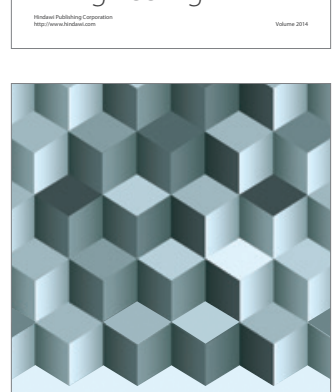

Journal of

Function Spaces
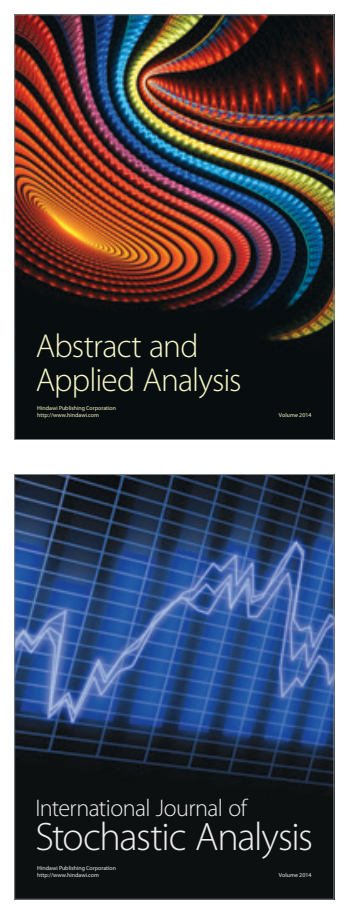

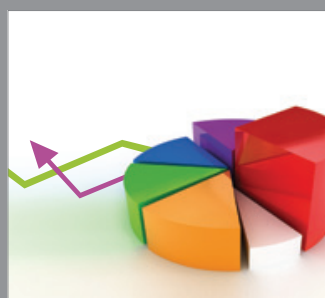

ournal of

Probability and Statistics

Promensencen
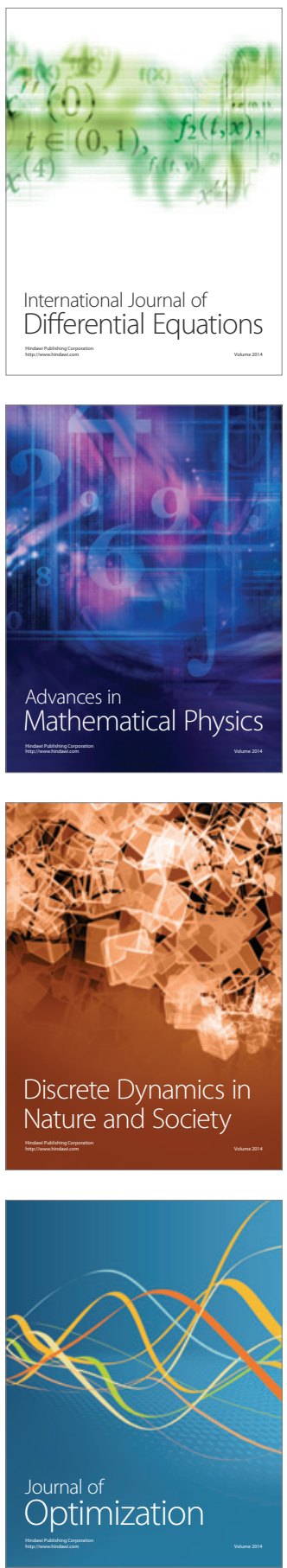